\title{
Proactive Adjustments of Response Strategies in the Stop-Signal Paradigm
}

\author{
Frederick Verbruggen \\ Vanderbilt University and Ghent University \\ Gordon D. Logan \\ Vanderbilt University

\begin{abstract}
In the stop-signal paradigm, fast responses are harder to inhibit than slow responses, so participants must balance speed on the go task with successful stopping in the stop task. In theory, participants achieve this balance by adjusting response thresholds for the go task, making proactive adjustments in response to instructions that indicate that relevant stop signals are likely to occur. The 5 experiments reported here tested this theoretical claim, presenting cues that indicated whether or not stop signals were relevant for the next few trials. Subjects made proactive response-strategy adjustments in each experiment: Diffusionmodel fits showed that response threshold increased when participants expected stop signals to occur, slowing go responses and increasing accuracy. Furthermore, the results show that subjects can make proactive response-strategy adjustments on a trial-by-trial basis, suggesting a flexible cognitive system that can proactively adjust itself in changing environments.
\end{abstract}

Keywords: response strategies, proactive control adjustments, stop-signal paradigm, reaction time models

Cognitive control processes are required to achieve a balance between competing goals in ever-changing environments (Baddeley, 1996; Logan, 1985; Miller \& Cohen, 2001). These control processes allow people to adjust response strategies in cognitively demanding situations. Response-strategy adjustments are typically investigated by manipulating task instructions (e.g., Howell \& Kreidler, 1963; Rinkenauer, Osman, Ulrich, Muller-Gethmann, \& Mattes, 2004), by presenting distracting information (e.g., Logan \& Zbrodoff, 1979, 1982), or by analyzing trials following an error or response conflict (e.g., Botvinick, Braver, Barch, Carter, \& Cohen, 2001; Gratton, Coles, \& Donchin, 1992; Rabbitt, 1966, 1968). In the present study, we investigated how people adjust response strategies in a multitasking situation know as the stopsignal paradigm (Lappin \& Eriksen, 1966; Logan \& Cowan, 1984; Vince, 1948). The stop-signal paradigm involves a trade-off between two tasks with opposing requirements: a go task, which requires subjects to respond as quickly as possible when a stimulus is presented, and a stop task, which requires subjects to stop the response when a stop signal is presented. Success on the go task (fast responding) implies failure on the stop task (not stopping a response); success on the stop task (stopping a response) implies failure on the go task (slow responding) In five experiments, we

Frederick Verbruggen, Department of Psychology, Vanderbilt University; and Department of Experimental Psychology, Ghent University, Ghent, Belgium; Gordon D. Logan, Department of Psychology, Vanderbilt University.

Frederick Verbruggen is a Postdoctoral Fellow of the Research Foundation-Flanders (FWO-Vlaanderen). This research was also supported by National Science Foundation Grant BCS 0446806, National Institute of Mental Health Grant R01-MH073879-01, and Air Force Office of Scientific Research Grant FA9550-07-1-0192 to Gordon D. Logan. We thank Andreas Voss for his support and Nachshon Meiran for helpful comments on a previous version of this paper.

Correspondence concerning this article should be sent to Frederick Verbruggen, Department of Psychology, Vanderbilt University, Nashville, TN 37203. E-mail: frederick.verbruggen@ugent.be examined whether subjects make proactive response-strategy adjustments when a precue informs them about the occurrence of stop signals on the next few trials, trading speed on the go task for success in the stop task.

In the stop-signal paradigm, subjects perform a go task and on a random selection of the trials (stop-signal trials), a stop signal is presented, instructing them to withhold their go responses. Several studies suggest that people change response strategies after stopsignal trials (e.g., Emeric et al., 2007; Rieger \& Gauggel, 1999; Schachar et al., 2004; Verbruggen \& Logan, in press; Verbruggen, Logan, Liefooghe, \& Vandierendonck, 2008). Rieger and Gauggel (1999) found that go reaction times (RTs) for no-stop-signal trials were prolonged when a stop signal was presented on the previous trial. They suggested that subjects change their response strategy after successful and unsuccessful inhibition to increase the probability of stopping on the next trial. Other researchers have found that response strategies changed only after unsuccessful stopping (Schachar et al., 2004; Verbruggen \& Logan, in press; Verbruggen, Logan, Liefooghe, et al., 2008). Schachar et al. and Verbruggen et al. suggested that subjects interpret responses on stop-signal trials as errors (see also, e.g., Rieger \& Gauggel, 1999; van Boxtel, van der Molen, \& Jennings, 2005) and that this leads to responsestrategy adjustments, a suggestion that is reminiscent of the common finding that subjects shift response thresholds after making errors in choice-response tasks (Rabbitt, 1966, 1968), trading speed for accuracy. These studies show that subjects make reactive response-strategy adjustments after stop-signal presentation (Rieger \& Gauggel, 1999) or after unsuccessful inhibition (Schachar et al., 2004; Verbruggen \& Logan, in press; Verbruggen, Logan, Liefooghe, et al., 2008). In the present study, we investigated whether subjects also make proactive response-strategy adjustments when a precue informs them that stop signals are likely on the next few trials.

In stop-signal experiments, subjects are told not to wait for the stop signal (e.g., Logan, Cowan, \& Davis, 1984; Logan, Schachar, \& Tannock, 1997; Ridderinkhof, Band, \& Logan, 1999; Rieger \& Gauggel, 1999; Verbruggen, Liefooghe, \& Vandierendonck, 
2004). Despite these instructions, RTs for no-stop-signal trials increase when stop-signal probability increases (e.g., Logan, 1981; Logan \& Burkell, 1986) and Verbruggen and colleagues found longer RTs in blocks in which stop signals were expected than in control blocks in which no stop signals were expected (Verbruggen, Liefooghe, Notebaert, \& Vandierendonck, 2005; Verbruggen et al., 2004; Verbruggen, Liefooghe, \& Vandierendonck, 2006; see also Rieger \& Gauggel, 1999; Stuphorn \& Schall, 2006).

These effects of the introduction of stop-signal trials are interpreted as evidence that subjects proactively change response strategies at the beginning of a stop-signal block, trading speed in the go task for success in the stop task (e.g., Logan \& Burkell, 1986). However, the longer RTs in stop-signal blocks could be due to increased cognitive demands: First, two task goals (going and stopping) are involved in stop-signal blocks (Logan \& Cowan, 1984; Verbruggen, Schneider, \& Logan, in press), whereas only one task goal (going) is involved in no-stop-signal blocks. Maintaining different task goals is thought to require cognitive control (Miller \& Cohen, 2001; Miyake et al., 2000), so increased cognitive demands could increase RT. Second, subjects have to monitor for stop signals while performing the go task in stop-signal blocks. Monitoring for auditory signals can slow responses to visual stimuli (e.g., Spence \& Driver, 1997; Vandierendonck, De Vooght, $\&$ Van der Goten, 1998b). Thus, dual-task requirements could also contribute to the RT differences between stop-signal blocks and no-stop-signal blocks.

We here report five experiments that examined how go performance is influenced by the presentation of a precue informing subjects about the occurrence of stop signals on the next few trials.
We distinguish between two hypotheses, the proactive-adjustment hypothesis and the dual-task requirement hypothesis. Alternatives to these hypotheses are discussed in the General Discussion.

The proactive-adjustment hypothesis assumes that subjects balance stopping and going by adjusting the response threshold in the go task. Performance in the stop-signal paradigm depends on the relative finishing times of a go process triggered by the go stimulus that races against a stop process triggered by the stop signal (Logan \& Cowan, 1984). If the stop process finishes before the go process, then subjects inhibit their response (signal-inhibit trials). If the go process finishes before the stop process, then subjects fail to inhibit their response (signal-respond trials). Increasing the response threshold will increase the finishing time of the go process and decrease the probability of responding given a stop signal, p(respondlsignal), which equals the probability of failing to inhibit. This trade-off is depicted in Figure 1. P(respondlsignal), F1 represented by the area under the curve to the left of the dashed line, depends primarily on three factors: RT, the delay between the target and the stop signal (stop-signal delay, or SSD), and the latency of the stop process (stop-signal reaction time, or SSRT). When SSD increases but SSRT and the RT distribution do not change, p(respondlsignal) increases (see Figure 1B vs. Figure 1A). When SSRT increases but SSD and the RT distribution do not change, p(respondlsignal) also increases (see Figure 1C vs. Figure 1A). However, when the RT distribution is shifted to the right but SSD and SSRT do not change, p(respondlsignal) decreases (see Figure 1D vs. Figure 1A). We assume that this third case-slowing RT without changing SSD or SSRT-is the result of proactive

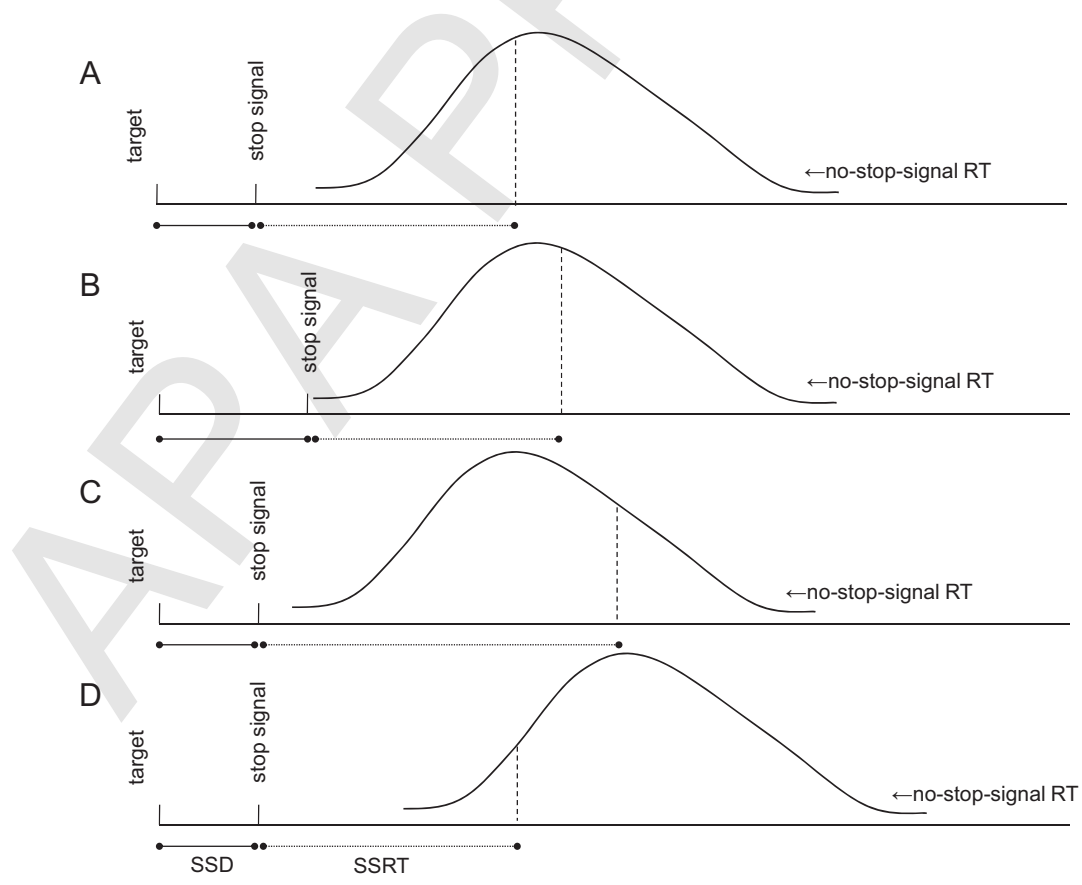

Figure 1. Illustration of the probabilities of responding, p(respondlsignal), based on the horse-race model (Logan \& Cowan, 1984), given the distribution of no-stop-signal reaction times (no-stop-signal RTs), the stop-signal delay (SSD), and the stop-signal reaction time (SSRT). P(respondlsignal) is represented by the area under the curve to the left of each dashed line. 
response-strategy adjustments that trade speed in the go task for success in the stop task.

The dual-task-requirements hypothesis assumes that the increased RTs are due to increased cognitive demands in stop-signal blocks: Stop-signal blocks involve two task goals (going and stopping), and subjects must attend to visual and auditory stimuli, whereas no-stop-signal blocks involve only one task goal (going), and subjects must attend only to visual stimuli. The increased working-memory load and the requirement to divide attention between audition and vision could slow RTs in stop-signal blocks. Thus, the major difference between the two hypotheses is that the proactive-adjustment hypothesis assumes that increased RTs are caused by a deliberate, proactive response-strategy shift, whereas the dual-task-requirements hypothesis assumes that increased RTs are caused by higher task demands in the stop-signal blocks.

The two hypotheses predict longer RTs for no-stop-signal trials when subjects expect a stop signal than when they do not. However, the two hypotheses make different predictions about accuracy. Models of RT assume that RT and accuracy are the results of a decision process in which information for the possible responses accumulates in response counters and that a response is chosen when the information in one of the counters exceeds a threshold (e.g., Ratcliff, 1978; Ratcliff, Van Zandt, \& McKoon, 1999; Usher $\&$ McClelland, 2001). The two hypotheses predict that the presence of stop signals will influence different parameters of the decision process (see Figure 2). The proactive-adjustment hypothesis predicts that the response threshold will be higher in stopsignal blocks than in no-stop-signal blocks. Increasing the thresh- old increases the amount of information required to choose a response, and that increases both RT and accuracy (Ratcliff, 1978). The dual-task-requirements hypothesis predicts that the presence of stop signals will increase the latency of nondecisional processes, such as stimulus encoding and motor execution, and lower the growth rate of information accumulation. Longer nondecision time and lower growth rate will both increase overall RT but will influence accuracy differently: Accuracy should be the same when nondecision time is influenced, whereas it should decrease when growth rate is influenced.

In the present study, we considered RT and accuracy together to distinguish between the proactive-adjustment hypothesis and the dual-task-requirement hypothesis. We also fitted the diffusion model to the data (Ratcliff, 1978; Ratcliff et al., 1999) so that we could attribute observed changes in speed and accuracy to specific underlying processes - threshold, drift rate, or decision time, alone or in combination (see Wagenmakers, van der Maas, \& Grasman, 2007). We fitted Fast-dm (Voss \& Voss, 2007), which allows fast and accurate estimations of the diffusion-model parameters even when the number of trials for every condition and subject is relatively low.

\section{The Present Study}

We conducted five experiments to determine whether subjects make proactive response-strategy adjustments in the stop-signal paradigm. In each experiment, subjects performed a choicereaction task and a stop signal was presented on a random selection

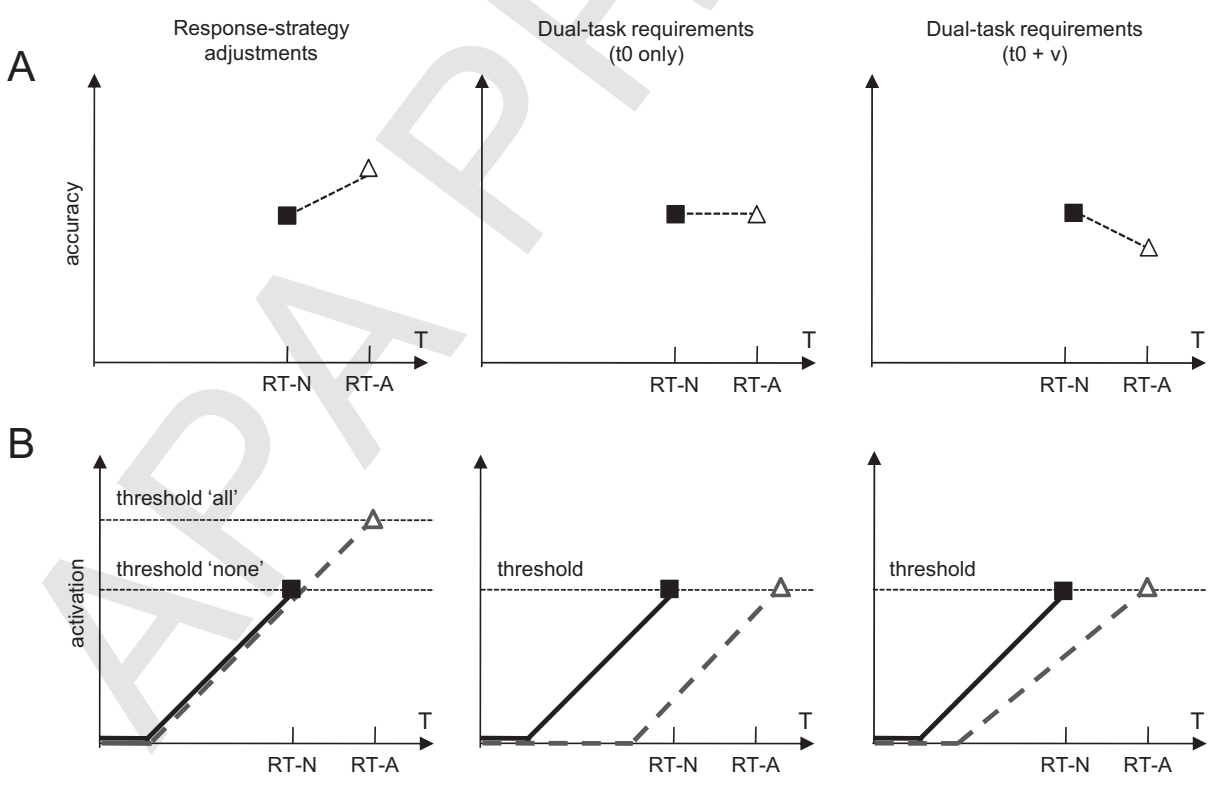

Figure 2. A: Reaction times (RTs; $x$-axis) and accuracy scores ( $y$-axis) predicted by the proactive-adjustment hypothesis (left chart) and the two versions of the dual-task requirements hypothesis in the none (N) and all (A) contexts. Version 1 (center chart): only nondecision time (t0) is influenced; Version 2 (right chart): nondecision time and growth rate (v) are influenced. B: Diffusion parameters (threshold, growth, and nondecision time) for the stop-signal contexts (none or all) predicted by the proactive-adjustment hypothesis (left chart) and the two versions of the dual-task requirements hypothesis (center and right charts). Growth rate corresponds to the slopes of the lines. Nondecision time $=$ stimulus processing + response execution. For purposes of clarity, we depicted total nondecision time as the time before the information starts to accumulate. 
of the trials. In addition, we presented context cues, making stop signals relevant or irrelevant for 4-8 trials within a block. In Experiment 1, we used two context cues: 'none', which indicated that none of the stop signals were relevant so they could be ignored, and 'all', which indicated that all stop signals were relevant and that subjects had to stop their response when a tone was presented.

In Experiment 1, we asked whether subjects use the precues to make response-strategy adjustments prior to the presentation of the go stimuli (they do). In Experiment 2, we asked whether dividing attention between the auditory and visual modality influenced performance, using a dual-task design in which subjects had to complete the first response and then execute a second response when a relevant tone occurred. We used two cues: 'single,' which indicated that all tones could be ignored, and 'dual,' which indicated that subjects had to press the space bar as quickly as possible when a tone occurred. Differences between the single and dual contexts assess the effects of dual-task requirements.

In Experiments 3-5, we focused only on proactive responsestrategy adjustments and examined how specific they are. In Experiment 3 , we manipulated the number of consecutive trials on which a cue remained on the screen to determine whether subjects proactively change response strategies when the cue changed after every trial. In Experiment 4, we prolonged the duration of the stop process by requiring subjects to inhibit selectively (e.g., Bedard et al., 2002; Riegler, 1986). We presented two new cues: 'low, which indicated that subjects were to stop their response only when a low tone was presented, and 'high,' which indicated that subjects were to stop their response only when a high tone was presented. To assess the effect on response-strategy adjustments, we compared performance with none and all cues. In Experiment 5 , we used cues that indicated the probability that a stop signal would be presented on the next trial (0\% stop signals, 30\% stop signals, or $70 \%$ stop signals) to determine whether stop-signal probability affects proactive response-strategy adjustments.

In all experiments, we compared the proactive-adjustment hypothesis to the dual-task requirements hypothesis. The proactiveadjustment hypothesis predicted higher response thresholds in stop-signal contexts, resulting in longer RTs and higher accuracy on no-stop-signal trials in stop-signal contexts (i.e., all and low/ high contexts, or the 30\% and 70\% contexts in Experiment 5) than in the none contexts. The dual-task-requirement hypothesis predicted longer nondecision time, lower growth rate, or both, in the stop-signal contexts, resulting in longer RTs and equivalent (when only nondecision processes change) or worse accuracy (when growth rate changes) in stop-signal contexts than in the none context.

\section{Experiment 1}

In Experiment 1, subjects discriminated between ' $\mathrm{Z}$ ' and ' $\mathrm{l}$ ' in the go task and stopped their response when they heard a relevant stop signal. The relevance of stop signals was indicated by context cues: 'none' indicated that none of the stop signals were relevant and could be ignored, whereas 'all' indicated that all stop signals were relevant so subjects had to stop their responses whenever a tone occurred.

Strayer and Kramer (1994) found that subjects could not change response criteria proactively in response to precues (see also
Brown \& Steyvers, 2005), but subjects were able to set response criteria at the beginning of each block and make reactive adjustments after each trial. These findings suggest that subjects may make proactive response-strategy adjustments only at the beginning of a stop-signal block (Logan, 1981; Logan \& Burkell, 1986; Verbruggen et al., 2004, 2006) and then make reactive adjustments after stop-signal trials (e.g., Rieger \& Gauggel, 1999; Verbruggen, Logan, Liefooghe, et al., 2008). Therefore, in Experiment 1, we asked whether subjects could use precues to adjust response criteria proactively.

We tested the proactive response-strategy adjustment hypotheses and the dual-task-requirement hypothesis by comparing nostop-signal performance in the none and all contexts. If subjects increase their response threshold to decrease p(respondlsignal), then RTs should be longer and accuracy should be higher in the all context than in the none context. If differences between the all and none context are due to dual-task requirements only, then RTs should be longer but accuracy should be similar or lower in the all context than in the none context, depending on whether nondecision or growth-rate parameters (or both) are influenced by the increased cognitive demands.

\section{Method}

Subjects. Eighteen students from Vanderbilt University participated for course credit. All subjects had normal or correctedto-normal vision and all were naive as to the purpose of the experiment.

Apparatus and stimuli. The experiment was run on a Pentium 4 PC running Tscope (Stevens, Lammertyn, Verbruggen, \& Vandierendonck, 2006). The stimuli were presented on a 21-in. cathode ray tube monitor. The go task was to respond to ' $\mathrm{Z}$ ' $(6 \times$ $9 \mathrm{~mm})$ or ' $\%(6 \times 9 \mathrm{~mm})$ by pressing the corresponding key of a QWERTY keyboard with the left and right index finger, respectively. The stop-signal context was indicated by the words 'none' $(25 \times 6 \mathrm{~mm})$ or 'all' $(20 \times 8 \mathrm{~mm})$. The stimuli and context cues were centrally presented in a white, lower case, Courier font on a black background. Context cues appeared $20 \mathrm{~mm}$ above the stimulus (see Figure 3). On signal trials, a loud and clear auditory F3 signal $(80 \mathrm{~dB}, 75 \mathrm{~ms})$ was presented through closed headphones (Sennheiser eH150), using stop-signal presentation functions of STOP-IT (Verbruggen, Logan, \& Stevens, 2008). One half of the

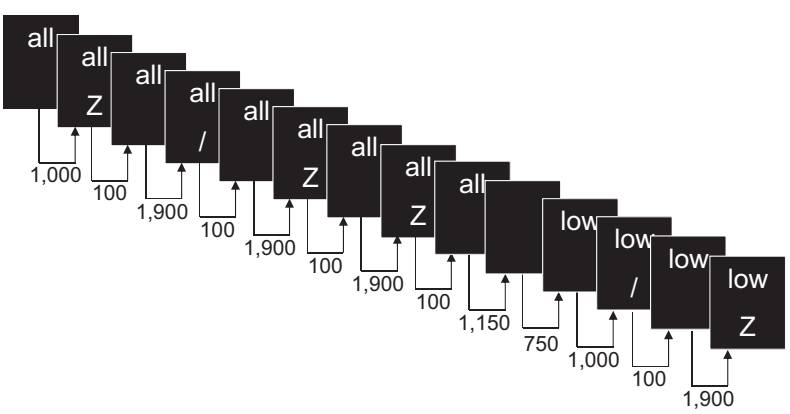

Figure 3. The sequence of events (from left to right). The numbers indicate the cue-presentation durations and stimulus-presentation durations (ms). 
signals were high tones $(750 \mathrm{~Hz})$, the other half were low tones $(250 \mathrm{~Hz})$.

Procedure. Instructions were explained orally by the experimenter. Instructions emphasized both accuracy and speed. Trials were divided in runs of 4 or 8 trials in the same context. Run length was randomized with the restriction that there were no context repetitions (e.g., $2 \times 8$ none context trials) and that runs of 4 trials and runs of 8 trials occurred with equal probability. Half of the trials were none context trials and half were all context trials.

All trial runs started with the presentation of the context cue (see Figure 3). After 1,000 ms, the first stimulus was presented. The stimulus was removed after $100 \mathrm{~ms}$ and required a response within $1,250 \mathrm{~ms}$. The interstimulus interval within the same context was $2,000 \mathrm{~ms}$, regardless of RT. The context cue remained on the screen during the whole run and was removed $1,250 \mathrm{~ms}$ after the presentation of the last stimulus of the run. The interval between the removal of the previous context cue and the presentation of the new cue was $750 \mathrm{~ms}$.

On one-third of the trials, a stop signal was presented. Half of the stop signals were high tones, the other half were low tones. In the none context, all stop signals were irrelevant and subjects were instructed to respond on all trials. In the all context, all stop signals were relevant and subjects were instructed to stop their response whenever a low or high tone was presented. Relevant and irrelevant stop signals were presented after a variable SSD. In the all context, the SSD was initially set at $150 \mathrm{~ms}$ and continuously adjusted according to a tracking procedure to obtain a probability of stopping of .50 (Logan et al., 1997). Each time a subject responded to the stimulus on a stop-signal trial, SSD decreased by $25 \mathrm{~ms}$. When subjects inhibited successfully, SSD increased by 25 $\mathrm{ms}$. Subjects were informed about this tracking procedure and they were told not to wait for a stop signal to occur. They were also told that it would be easy to stop on some trials and difficult or impossible to stop on other trials because SSD varied substantially. In the none context, the SSD was the same as the current SSD for the all context trials.

The experiment started with a practice block of 24 trials without stop signals. This no-stop-signal block was followed by a practice block of 48 trials in which the context cues and both relevant and irrelevant stop signals were presented. During the practice block, subjects received immediate feedback at the end of each trial. On no-stop-signal trials, 'wrong' appeared when they made an error and 'try to respond faster' appeared when they did not respond in time. On irrelevant stop-signal trials, 'this was an irrelevant signal' was presented when they erroneously stopped their response. On relevant stop-signal trials, 'this was a relevant signal' was presented when subjects responded. The feedback remained in the center of the screen for $750 \mathrm{~ms}$. The experimental phase consisted of 6 blocks of 96 trials. At the end of each block, we presented the number of no-stop-signal errors, the mean RT, the number of incorrectly stopped responses in the none context, and the probability of stopping in the all context. Subjects had to pause for 10 seconds.

\section{Results and Discussion}

Because go performance is influenced by stop-signal presentation or erroneous go responses on the previous trials (e.g., Rabbitt, 1966; Rieger \& Gauggel, 1999; Verbruggen, Logan, Liefooghe, et al., 2008), we excluded trials that followed a stop-signal trial or an incorrect no-stop-signal trial (including no-stop-signal trials on which no response was executed) in analyses ${ }^{1}$ of go performance. Fn1

Mean RTs were calculated after removal of go errors. Outlying RTs (RTs longer than $2.5 \mathrm{SDs}$ above the mean for each trial type; $2.9 \%$ of the trials) were discarded from data analysis. Proactive response-strategy adjustments could result in a higher percentage of omitted responses as well as higher accuracy, so we distinguished between omission rate and accuracy: omission rate is the ratio of the number of omitted responses to the total number of no-stop-signal trials-that is, omission rate $=100 \times$ omitted/(correct + incorrect + omitted)-and accuracy is the ratio of the number of correct responses to the number of correct and incorrect responses-that is, accuracy $=100 \times$ correct/(correct + incorrect).

Signal analyses. P(respondlsignal), SSD, and SSRT are presented in Table 1. We compared go performance on stop-signal T1 trials with go performance on no-stop-signal trials in the none and all contexts by means of separate repeated measures analyses of variance (ANOVAs). RT, accuracy, and omission rate are presented in Table 2. A summary of the ANOVAs is presented in $T 2$ Table 3.

In the none context, RT was longer for stop-signal trials (425 $\mathrm{ms})$ than for no-stop-signal trials $(408 \mathrm{~ms})$. Omission rate was higher for stop-signal trials $(2.4 \%)$ than for no-stop-signal trials $(0.9 \%)$. These findings suggest that subjects could not completely ignore irrelevant stop signals. Accuracy was comparable for stopsignal trials and no-stop-signal trials. In the all context, we found that RTs were shorter for signal-respond trials $(444 \mathrm{~ms})$ than for no-stop-signal trials $(501 \mathrm{~ms})$, which is consistent with the horserace model of stop-signal performance (Logan \& Cowan, 1984). Accuracy was comparable for signal-respond and no-stop-signal trials.

Context analyses. We analyzed RTs, accuracy data, and omission rates for no-stop-signal trials by means of repeated measures ANOVAs with context as a within-subjects factor. A summary of the ANOVAs is presented in Table 4. Observed RTs and accuracy T4 scores are depicted in Figure 4A; standard deviations are presented F4 in Table 2. We also examined the effect of trial position following the context cues (see Figure 3); these position analyses appear in Appendix A.

Consistent with the proactive adjustment hypothesis, we found significantly longer RTs (all $=501 \mathrm{~ms}$; none $=408 \mathrm{~ms}$ ) and higher accuracy (all $=98.7 \%$; none $=97.2 \%$ ) in the all context than in the none context, suggesting that the response threshold was increased in the all context. The percentage of omitted responses was similar in the all and none contexts.

\footnotetext{
${ }^{1}$ Results of Emeric et al. (2007) suggest that stop-signal presentation on trial $n-2$ did not influence no-stop-signal performance on trial $n$ (but see Verbruggen \& Logan, in press, for long-term aftereffects of stopping when large stimulus sets are used). Consistent with Emeric et al.'s finding, we found similar results when we included all trials that followed a no-stopsignal trial (i.e., trial $n-1$ was a no-stop-signal trial) and when we included only trials that followed at least two no-stop-signal trials (i.e., trial $n-1$ and trial $n-2$ were no-stop-signal trials), suggesting that there was no effect of stop-signal presentation on trial $n-2$. Given this finding, we included all trials that immediately followed a no-stop-signal trial to maximize the number of observations.
} 


\begin{tabular}{|l|l|l|l|l|l|l|}
\hline tapraid5/zfn-xhp/zfn-xhp/zfn00608/zfn2273d08z & royerl & $\mathrm{S}=3$ & $7 / 8 / 08$ & $10: 29$ & Art: 2007-0917 \\
\hline
\end{tabular}

Table 1

Relevant Stop-Signal Data (SE in Parentheses) for Experiments $1-5$

\begin{tabular}{lccc}
\hline $\begin{array}{c}\text { Experiment and } \\
\text { context }\end{array}$ & P(respond signal) & SSD (in ms) & SSRT (in ms) \\
\hline $\begin{array}{l}\text { Experiment 1 } \\
\text { Experiment 3, run }\end{array}$ & $.47(.01)$ & $247(26)$ & $263(17)$ \\
$\quad$ length 1 & $.49(.01)$ & $353(46)$ & $223(19)$ \\
$\begin{array}{l}\text { Experiment 3, run } \\
\quad \text { length 2 }\end{array}$ & $.49(.01)$ & $351(58)$ & $233(17)$ \\
$\begin{array}{l}\text { Experiment 3, run } \\
\text { length 4 }\end{array}$ & $.51(.02)$ & $298(41)$ & $255(11)$ \\
$\begin{array}{l}\text { Experiment 4, } \\
\text { low/high }\end{array}$ & $.49(.02)$ & $181(24)$ & $259(14)$ \\
Experiment 4, all & $.47(.02)$ & $205(22)$ & $229(10)$ \\
Experiment 5, 30\% & $.49(.01)$ & $269(17)$ & $226(10)$ \\
Experiment 5, 70\% & $.49(.01)$ & $408(28)$ & $213(13)$ \\
\hline
\end{tabular}

Note. We used the integration method to calculate stop-signal reaction time (SSRT; Logan \& Cowan, 1984). The no-stop-signal reaction times (RTs) are rank-ordered, and the $n$th RT is selected, where $n$ is obtained by multiplying the number of RTs in the distribution by the probability of responding at a given delay. To estimate SSRT, stop-signal delay (SSD) is subtracted from the $n$th RT. This process is repeated for each SSD for each subject. The results are then averaged across SSDs. However, when using the tracking procedure, some SSDs will occur more often than other SSDs. In order to obtain reliable SSRT estimations, we selected for each subject the two SSDs that occurred most frequently and we calculated SSRT for both SSDs separately. In a final step, we took for each subject the average of both SSRTs. P(respond signal) = the probability of responding given a stop signal.

Diffusion-model fits. We used Fast- $\mathrm{dm}^{2}$ to estimate the parameters of the diffusion model for each subject. Boundary separation ( $a$; i.e., the distance between the decision thresholds; larger values of $a$ result in fewer errors but longer RTs), growth rate ( $v$; larger values of $v$ result in fewer errors and shorter RTs), and nondecision time ( $t 0$; larger values of $t 0$ result in longer RTs) were allowed to take different values in the none context and the all context. We estimated the parameters for each subject and each stop-signal context separately and examined the effect of stop-signal context on the parameters in separate repeated-measures ANOVAs with context as a withinsubjects factor. The mean values for each parameter are presented in Table 5, the estimated decision time and nondecision time are depicted in Figure 4B, and the analyses appear in Table 6.

Consistent with the proactive-adjustment hypothesis, we found a significant increase in boundary separation in the all context (all = 1.30 , none $=1.12$ ), demonstrating that subjects increased response thresholds when they expected relevant stop signals to occur. (The model fits assumed symmetrical boundaries, so response threshold $=$ boundary separation/2.) We also found a significant increase in nondecision time (all $=327 \mathrm{~ms}$, none $=295 \mathrm{~ms}$ ) and a decrease in growth rate (all $=3.76$ units $/ \mathrm{sec}$, none $=4.45$ units $/ \mathrm{sec}$ ), which is consistent with the dual-task requirement hypothesis.

Summary. Consistent with the proactive-adjustment hypothesis, the behavioral results and the estimated parameters suggest that subjects increased response threshold when they expected stop signals to occur on the next few trials. The position analyses presented in Appendix A suggest that subjects made these adjustments at the beginning of the all context. However, the differences in nondecision time and growth rate suggest that dual-task requirements also contributed to the difference between the all and none contexts. We tested this hypothesis in Experiment 2.

\section{Experiment 2}

In Experiment 2, we tested the effects of dual-task requirements directly by having subjects perform a second go task on relevant signal trials. The primary go task (the gol task) was the same as in Experiment 1 . However, subjects no longer had to stop the response when a relevant signal was presented. Instead, they had to execute a second response. We used two cues: 'single,' which indicated that all tones could be ignored, and 'dual,' which indicated that subjects had to press the space bar as quickly as possible on signal trials (i.e., perform a go 2 task) after they executed the go1 response. The dual context required subjects to maintain two task goals (the go1 goal and the go2 goal) and monitor for dual-task signals. Differences in go1 RT and accuracy between the single and dual contexts would support the dual-task requirements hypothesis. A pattern of differences similar to the pattern observed in Experiment 1 would suggest that dual-task requirements may also limit performance in the stop-signal paradigm.

\section{Method}

Subjects. Eighteen students from Vanderbilt University participated for course credit. All subjects had normal or corrected-tonormal vision and all were naive as to the purpose of the experiment.

Apparatus, stimuli, and procedure. These were the same as in Experiment 1 except for the following: Half of the trials were single context trials and the other half were dual context trials; the contexts were indicated by the cues 'single' and 'dual,' respectively. In the single context, subjects could ignore all tones. In the dual context, subjects had to press the space bar with the index fingers of both hands when a tone was presented. Subjects were instructed to execute the ' $\mathrm{Z}$ ' or ' $/$ ' response (the gol responses) first and grouping of responses was discouraged. We simulated a tracking procedure to produce a range of SSDs similar to the ones observed in Experiment 1: When go1 RT on a dual-task trial was longer than SSD $+263 \mathrm{~ms}$ (the mean SSRT in Experiment 1), SSD decreased by $25 \mathrm{~ms}$ (viz., signal-respond); when gol RT on a dual-task trial was shorter than SSD $+263 \mathrm{~ms}$, SSD increased by 25 ms (viz., signal-inhibit).

\section{Results and Discussion}

As in Experiment 1, we excluded trials that followed a dual-task trial or an incorrect no-signal trial in analyses of gol performance. Mean go1 RTs were calculated after removal of gol errors and outlying RTs ( $2.1 \%$ of the trials).

Signal analyses. Go1 RT, accuracy, and omission rate are presented in Table 2. A summary of the ANOVAs is presented in Table 3. In the single context, subjects pressed the space bar on $6.3 \%(S E=1.3 \%)$ of the signal trials, which is significantly different from $0 \%, t(17)=5.1, p<.001$. No subjects pressed the

\footnotetext{
${ }^{2}$ No-stop-signal trials following correct no-stop-signal trials were coded as correct or incorrect. For the model fits, starting point $(z)$ was set to 0.5 (when responses are coded as correct or incorrect, it is implausible that $\mathrm{z}$ deviates from $a / 2$; therefore, $z$ - which refers to a fraction of the boundary separation-was fixed at 0.5$)$. Boundary separation $(a)$, growth rate $(v)$, and nondecision time $(t O)$ were allowed to take different values in the different stop-signal contexts.
} 
Table 2

Reaction Time (RT, in ms), Accuracy (\%), and Omission Rate (\%) for the Different Stop-Signal Contexts in Experiments 1-5 (SDs in Parentheses)

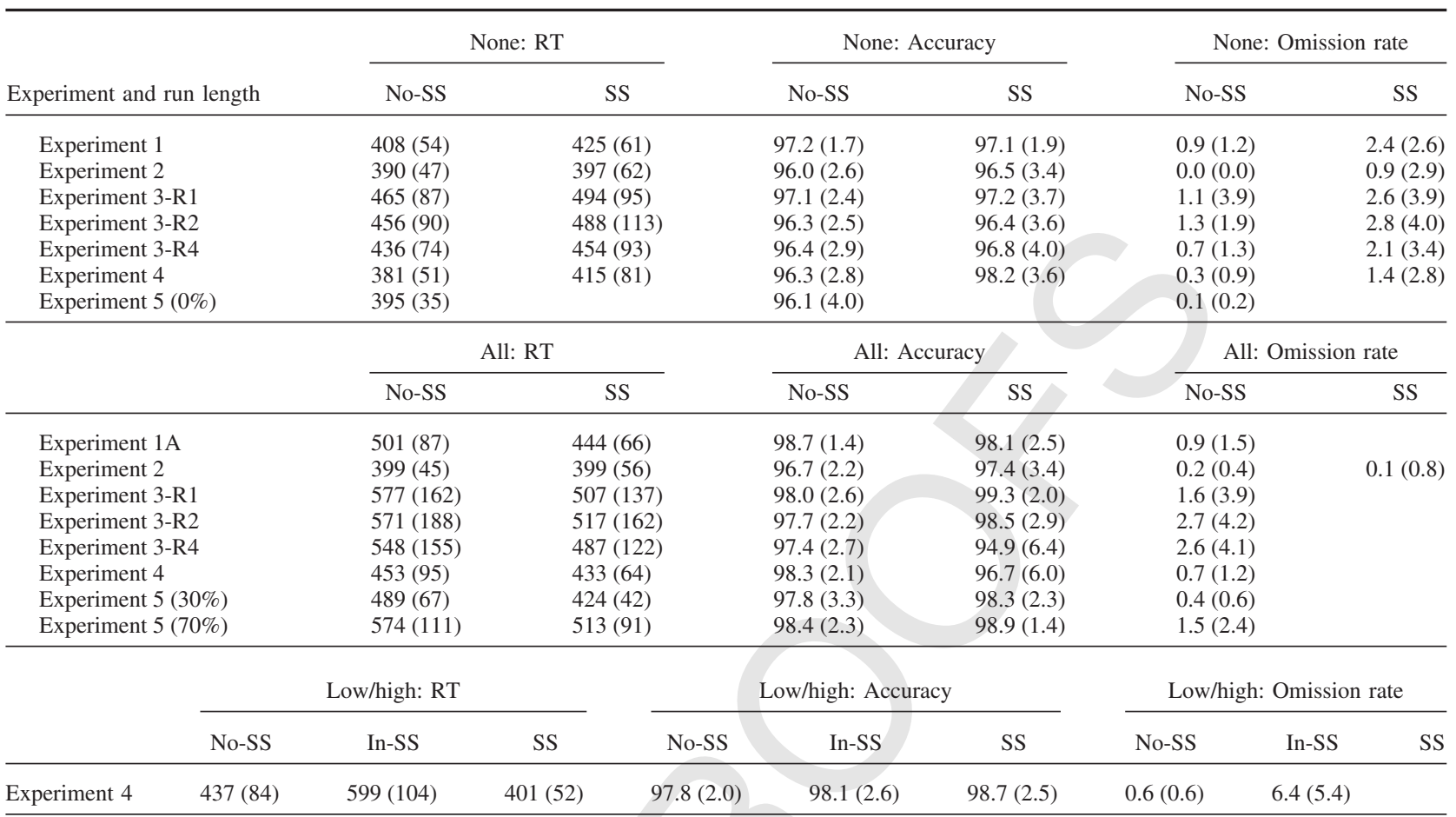

Note. In Experiment 2, the single context corresponds to the none context; the dual context corresponds to the all context. In Experiment 5, the 0\% context corresponds to the none context. No-SS = no-stop-signal trial; SS = stop-signal trial; In-SS = invalid-stop-signal trial; R1 = run length 1 ; R2 = run length $2 ; \mathrm{R} 4$ = run length 4.

space bar on no-signal trials. Go1 RT and accuracy were comparable for no-signal and signal trials. Omission rate for no-signal trials was $0 \%$; omission rate for signal trials $(0.2 \%)$ was not significantly different from $0 \%, t(1,17)=1.18, p>.18$. In the dual context, subjects pressed the space bar on $97.1 \%(S E=0.6 \%)$ of the signal trials. Mean stimulus-onset asynchrony was $149 \mathrm{~ms}$ $(S E=13 \mathrm{~ms})$ and go2 RT was $558 \mathrm{~ms}(S E=11 \mathrm{~ms})$. Go1 RT, accuracy and omission rate were comparable for no-signal and signal trials, suggesting that subjects did not group responses. No subjects pressed the space bar on no-signal trials.

Context analyses. Go1 RTs and accuracy were analyzed by means of repeated measures ANOVAs with context as a withinsubject factor. A summary of the ANOVAs is presented in Table 4. Observed go1 RTs and accuracy scores are depicted in Figure 4A; standard deviations appear in Table 2.

To test the effects of dual-task requirements, we compared performance in the dual and single contexts. Go1 RTs for no-signal trials were significantly longer in the dual context $(399 \mathrm{~ms})$ than in the single context $(390 \mathrm{~ms})$. There was no effect of context on accuracy. These findings suggest that subjects did not make proactive response-strategy adjustments when they expected dual-task signals to occur. The small RT difference between the single and dual contexts is probably due to the dual-task requirements. Importantly, the RT difference was smaller than in Experiment 1, suggesting that the RT difference in Experiment 1 was mostly due to proactive response-strategy adjustments. Omission rate was $0 \%$ in the single context and $0.2 \%$ in the dual context. The latter difference was significantly greater than $0 \%, t(1,17)=2.2, p<$ .05 . Because subjects did not make proactive response-strategy adjustments, we did not analyze the data as a function of position.

Diffusion-model fits. As in Experiment 1, we used Fast-dm to estimate the diffusion parameters for each subject. The mean values for each parameter are presented in Table 5, and the estimated decision time and nondecision time are depicted in Figure 4B; the analyses appear in Table 6. In contrast to Experiment 1, we did not find a significant difference in boundary separation (dual $=.93$, single $=.93$ ). Consistent with the dual-task requirements hypothesis, we found that nondecision time was significantly longer in dual-task (309 ms) than in single-task (295 $\mathrm{ms}$ ) conditions. Paradoxically, growth rate was significantly higher in the dual-task condition (5.03 units/sec) than in the single-task condition (4.59 units/sec).

These fits suggest that diffusion-model parameters are influenced differently by introducing stop signals (Experiment 1) and dual-task signals (Experiment 2). To further test the difference between Experiments 1 and 2, we reanalyzed diffusion-model parameters by means of a mixed ANOVA with context (none/ single vs. all/dual) as a within-subjects factor and experiment (Experiment 1 vs. Experiment 2) as a between-subjects factor. We will focus on the interaction between the two factors only. 
Table 3

Summary of Analyses of Variance Performed to Compare Go Performance on No-Stop-Signal and Signal Trials (dfs in Parentheses)

\begin{tabular}{|c|c|c|c|c|c|c|c|c|c|}
\hline \multirow[b]{2}{*}{ Experiment and context } & \multicolumn{3}{|c|}{$\mathrm{RT}$} & \multicolumn{3}{|c|}{ Accuracy } & \multicolumn{3}{|c|}{ Omitted responses } \\
\hline & $F$ & $M S E$ & $\eta_{p}^{2}$ & $F$ & MSE & $\eta_{p}^{2}$ & $F$ & $M S E$ & $\eta_{p}^{2}$ \\
\hline \multicolumn{10}{|l|}{ Experiment 1} \\
\hline None $(1,17)$ & $5.2^{*}$ & 519 & .24 & 0.0 & 3.0 & .00 & $5.7^{*}$ & 3.5 & .25 \\
\hline All $(1,17)$ & $33.3^{* *}$ & 859 & .66 & 1.3 & 2.4 & .07 & & & \\
\hline \multicolumn{10}{|l|}{ Experiment 2} \\
\hline Single $(1,17)$ & 1.5 & 377 & .08 & 0.5 & 4.6 & .03 & & & \\
\hline Dual $(1,17)$ & 0.0 & 335 & .00 & 1.3 & 2.7 & .07 & 0.7 & 0.1 & .04 \\
\hline \multicolumn{10}{|l|}{ Experiment 3} \\
\hline None $(1,17)$ & $14.7^{* * *}$ & 1,297 & .46 & 0.2 & 5.0 & .01 & $13.5^{* * *}$ & 4.3 & .44 \\
\hline All $(1,17)$ & $32.0^{* *}$ & 3,188 & .65 & 0.0 & 10.0 & .00 & & & \\
\hline \multicolumn{10}{|l|}{ Experiment 4} \\
\hline None $(1,17)$ & $6.6^{*}$ & 1,503 & .28 & 2.5 & 14.0 & .13 & 2.4 & 4.5 & .12 \\
\hline All $(1,17)$ & 1.4 & 2,637 & .08 & 1.0 & 21.7 & .06 & & & \\
\hline $1 / \mathrm{h}(2,34)$ & $87.5^{* *}$ & 2,280 & .84 & 0.9 & 4.0 & .05 & $21.7^{* * *}$ & 14.0 & .56 \\
\hline \multicolumn{10}{|l|}{ Experiment 5} \\
\hline $30 \%(1,17)$ & $41.9^{* * *}$ & 911 & .71 & 1.2 & 2.0 & .07 & & & \\
\hline $70 \%(1,17)$ & $46.4^{*}$ & 718 & 73 & 1.3 & 1.8 & .07 & & & \\
\hline
\end{tabular}

Note. For the analyses of omitted responses in the low/high context, we included only no-stop-signal trials and invalid-signal trials $(d f=1,17) .1 / \mathrm{h}=$ low/high context; $30 \%=30 \%$ context, $70 \%=70 \%$ context.

${ }^{*} p<.05 .{ }^{* *} p<.01$.

For boundary separation, we found a significant interaction between context and experiment (see Table 6), suggesting that subjects increased the response threshold in the all context but not in the dual context. (Note that boundary separation was higher overall in Experiment 1 than in Experiment 2). The interaction between context and experiment was also significant for growth rate: Drift rate was lower in the all context than in the none context whereas it was higher in the dual context than in the single context. This finding suggests that the growth-rate difference in Experiment 1 was not due to dual-task requirements. The nondecision time difference was larger in Experiment 1 (32 ms) than in Experiment 2 (14 ms), but the interaction did not reach significance. We will discuss the growth-rate and nondecision time differences further in the General Discussion.
Summary. The behavioral results and the diffusion-model fits of Experiment 2 suggest that subjects did not increase the response threshold when they expected dual-task signals to occur on the next few trials. This finding further supports the hypothesis that response-threshold adjustments in Experiment 1 were specifically due to the introduction of stop signals: Stop performance benefited from slow go1 RTs whereas go2 performance benefited from fast go2 RTs. However, we did find RT differences between the single and dual context, suggesting that dual-task requirements also influenced performance. This finding was further supported by the significant differences in nondecision time in the diffusion-model fits. Thus, the results of Experiment 2 suggest that the RT differences between the none context and the all context in Experiment

Table 4

Summary of Analyses of Variance Performed to Test the Main Effect of Context in Experiments 1-5 (dfs in Parentheses)

\begin{tabular}{|c|c|c|c|c|c|c|c|c|c|}
\hline \multirow[b]{2}{*}{ Experiment and factor } & \multicolumn{3}{|c|}{ RT } & \multicolumn{3}{|c|}{ Accuracy } & \multicolumn{3}{|c|}{ Omitted responses } \\
\hline & $F$ & $M S E$ & $\eta_{p}^{2}$ & $F$ & $M S E$ & $\eta_{p}^{2}$ & $F$ & $M S E$ & $\eta_{p}^{2}$ \\
\hline Experiment $1(1,17)$ & $28.4^{* * *}$ & 2,728 & .63 & $18.4^{* *}$ & 1.1 & .52 & 0.0 & 0.7 & .00 \\
\hline Experiment $2(1,17)$ & $6.0^{*}$ & 119 & .26 & 1.9 & 2.4 & .10 & & & \\
\hline \multicolumn{10}{|l|}{ Experiment $3(1,17)$} \\
\hline $\mathrm{C}(1,17)$ & $21.2^{* * *}$ & 16273 & .56 & $16.3^{* * *}$ & 2.0 & .49 & $4.6^{*}$ & 8.6 & .21 \\
\hline $\operatorname{RL}(2,34)$ & 1.8 & 4,674 & .10 & 1.2 & 4.1 & .06 & 0.3 & 13.6 & .02 \\
\hline $\mathrm{C} \times \mathrm{RL}(2,34)$ & 0.0 & 3,030 & .00 & 0.2 & 3.6 & .01 & 1.6 & 2.7 & .09 \\
\hline Experiment $4(2,34)$ & $14.3^{* *}$ & 1,778 & .46 & 6.4 & 3.2 & .27 & 1.2 & 0.7 & .07 \\
\hline PC: n vs. $\operatorname{lh}(1,34)$ & $15.7^{* * *}$ & 1,778 & .32 & $6.9^{*}$ & 3.2 & .17 & & & \\
\hline PC: $n$ vs. a $(1,34)$ & $26.6^{* *}$ & 1,778 & .44 & $11.8^{* * *}$ & 3.2 & .17 & & & \\
\hline PC: lh vs. a $(1,34)$ & 1.3 & 1,778 & .04 & 0.7 & 3.2 & .02 & & & \\
\hline Experiment $5(2,34)$ & $40.1^{* *}$ & 3,581 & .70 & $4.6^{*}$ & 5.2 & .21 & $4.5^{*}$ & 2.2 & .21 \\
\hline PC: $0-30(1,34)$ & $22.3^{* * *}$ & 3,581 & .40 & $4.7^{*}$ & 5.2 & .12 & 0.5 & 2.2 & .02 \\
\hline PC: $0-70(1,34)$ & $80.2^{* * *}$ & 3,581 & .70 & $8.5^{* * *}$ & 5.2 & .20 & $8.2^{* * *}$ & 2.2 & .19 \\
\hline PC: $30-70(1,34)$ & $18.0^{* * *}$ & 3,581 & .34 & 0.6 & 5.2 & .02 & $4.7^{*}$ & 2.2 & .12 \\
\hline
\end{tabular}

Note. $\mathrm{C}=$ context $\mathrm{RL}=$ run length; $\mathrm{PC}=$ planned comparison; $\mathrm{n}=$ none context; $1 / \mathrm{h}=$ low/high context; $\mathrm{a}=$ all context.

${ }^{*} p<.05 . \quad{ }^{* *} p<.01$. 
A

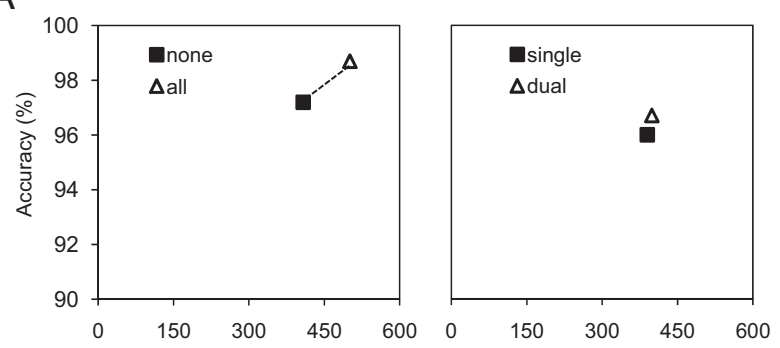

B

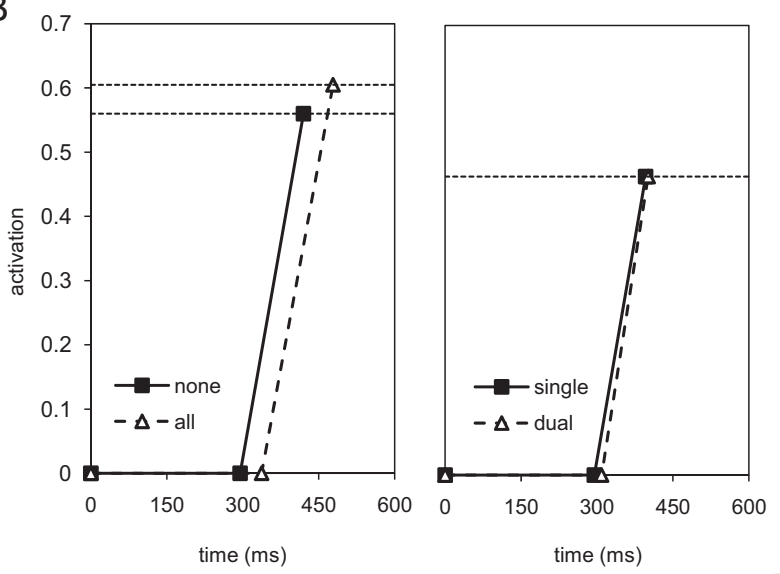

Figure 4. A: Observed reaction times ( $x$-axis) and accuracy ( $y$-axis) for each context for Experiments 1 and 2. B: Estimated decision and nondecision time for each context for Experiments 1 and 2. Growth rate $=$ accumulated evidence per second and response threshold (dotted lines) $=$ boundary separation / 2. Decision time $=$ response threshold / growth rate. Nondecision time $=$ stimulus processing + response execution. For purposes of clarity, we depict total nondecision time as the time before the information starts to accumulate.

1 were due to both proactive response-strategy adjustments and dual-task requirements.

\section{Experiment 3}

The results of Experiment 1 suggest that subjects made proactive response-strategy adjustments when they expected relevant stop signals to occur. The position analyses presented in Appendix A suggest that subjects make these adjustments at the beginning of the all context. These findings are inconsistent with the results of Strayer and Kramer (1994) and suggest that subjects can adjust response criteria proactively in response to precues in the stopsignal paradigm. One explanation for the discrepancy between our findings and those of Strayer and Kramer (1994) is that we used longer runs of trials, which may have encouraged subjects to make proactive adjustments. We tested this hypothesis in Experiment 3 by manipulating run length between blocks of trials. There were three run-length conditions: run length 1 , in which, the cue changed on every trial, run length 2 , in which the cue changed every two trials, and run length 4 , in which the cue changed every four trials. The go task and the stop-signal contexts were the same as in Experiment 1. If subjects make proactive response-strategy adjustments only when the context remains the same for several trials, then there should be no difference between the all and none contexts for run length 1 and, possibly, a smaller difference for run length 2 than for run length 4 .

Subjects. Eighteen students from Vanderbilt University participated for course credit. All subjects had normal or correctedto-normal vision and all were naive as to the purpose of the experiment.

Apparatus, stimuli, and procedure. These were the same as in Experiment 1 except for the following: There were three runlength conditions: run length 1 (context changes on every trial), run length 2 (context changes every two trials), and run length 4 (context changes every four trials). The order of the conditions was counterbalanced. Every run-length condition consisted of a practice phase with stop signals ( 24 trials) and an experimental phase, which consisted of three blocks of 96 trials.

\section{Results and Discussion}

Exclusion criteria were the same as in Experiment 1. Mean RT for included trials was calculated after exclusions of outliers $(2.1 \%)$.

Signal analyses. P(respondlsignal), mean SSD, and mean SSRT are presented in Table 1. As can be seen, run length did not affect stop-signal performance (all $p \mathrm{~s}>.19$ ). RT, accuracy, and omission rate are presented in Table 2. In the initial analyses, the main effect of signal did not interact with run length. Therefore, we collapsed the data across the different run lengths for the comparison of no-stop-signal and stop-signal trials. The analyses of the main effect of signal are presented in Table 3.

In the none context, RT was longer for stop-signal trials (479 $\mathrm{ms}$ ) than for no-stop-signal trials $(452 \mathrm{~ms})$. Omission rate was higher for stop-signal trials $(2.5 \%)$ than for no-stop-signal trials $(1.0 \%)$. These findings are consistent with the findings of Experiment 1 and suggest that subjects could not completely ignore irrelevant stop signals. Accuracy was comparable for stop-signal and no-stop-signal trials. In the all context, signal-respond RTs (504 ms) were shorter than no-stop-signal RTs $(565 \mathrm{~ms})$, as predicted by the race model (Logan \& Cowan, 1984); accuracy was comparable for signal and no-stop-signal trials.

Context analyses. To determine whether run length influenced proactive response-strategy adjustments, we analyzed no-stopsignal performance by means of 2 (context: none or all) $\times 3$ (run length: 1, 2, or 4) repeated measures ANOVAs. A summary of the ANOVAs is presented in Table 4. Observed RTs and accuracy scores are depicted in Figure 5A; standard deviations appear in F5 Table 2. We again analyzed the data as a function of position in the run of trials; these position analyses appear in Appendix B.

Overall, we found significantly longer RTs (all $=565 \mathrm{~ms}$, none $=452 \mathrm{~ms}$ ) and higher accuracy (all $=97.7 \%$, none $=96.6 \%$ ) in the all context than in the none context. These findings suggest that subjects increased the response threshold in the all context, trading speed in the go task for success in the stop task. Omission rate was also higher in the all context $(2.3 \%)$ than in the none context $(1.0 \%)$. We did not find a main effect of run length nor an interaction between context and run length, suggesting that subjects made similar proactive response-strategy adjustments in the three run lengths. 
Table 5

Estimated Diffusion Parameters for Experiments 1-5 (SD in Parentheses)

\begin{tabular}{|c|c|c|c|c|c|c|c|}
\hline Experiment and context & $a$ & $v$ & $t 0(\mathrm{~ms})$ & $s z$ & sv & sto & $p$ \\
\hline \multicolumn{8}{|l|}{ Experiment 1} \\
\hline All & $1.30(.24)$ & $3.76(.77)$ & $327(49)$ & \multirow{2}{*}{$.37(.12)$} & \multirow[t]{2}{*}{$.31(.32)$} & \multirow{2}{*}{$.15(.05)$} & \multirow{2}{*}{$.74(.26)$} \\
\hline None & $1.12(.20)$ & $4.45(1.08)$ & $295(33)$ & & & & \\
\hline \multicolumn{8}{|l|}{ Experiment 2} \\
\hline Dual & $0.93(.20)$ & $5.03(1.07)$ & $309(23)$ & \multirow[t]{2}{*}{$.27(.07)$} & \multirow[t]{2}{*}{$.50(.97)$} & \multirow[t]{2}{*}{$.14(.05)$} & \multirow[t]{2}{*}{$.90(.10)$} \\
\hline Single & $0.93(.16)$ & $4.59(1.09)$ & $295(23)$ & & & & \\
\hline \multicolumn{8}{|l|}{ Experiment 3: Run length 1} \\
\hline All & $1.27(.31)$ & $3.50(1.04)$ & 387 (109) & \multirow[t]{2}{*}{$.41(.16)$} & $.22(.18)$ & \multirow[t]{2}{*}{$.17(.10)$} & \multirow[t]{2}{*}{$.87(.14)$} \\
\hline None & $1.18(.27)$ & $3.80(.97)$ & $312(42)$ & & & & \\
\hline \multicolumn{8}{|l|}{ Experiment 3: Run length 2} \\
\hline All & $1.23(.31)$ & $3.47(1.21)$ & $397(142)$ & \multirow[t]{2}{*}{$.35(.16)$} & $.32(.20)$ & \multirow[t]{2}{*}{$.15(.08)$} & \multirow[t]{2}{*}{$.82(.22)$} \\
\hline None & $1.12(.26)$ & $3.67(1.02)$ & $319(40)$ & & & & \\
\hline \multicolumn{8}{|l|}{ Experiment 3: Run length 4} \\
\hline All & $1.17(.24)$ & $3.50(1.10)$ & $385(96)$ & \multirow{2}{*}{$.34(.11)$} & $.25(.15)$ & \multirow{2}{*}{$.16(.06)$} & \multirow[t]{2}{*}{$.83(.22)$} \\
\hline None & $1.12(.18)$ & $3.74(.68)$ & $301(50)$ & & & & \\
\hline \multicolumn{8}{|l|}{ Experiment 4} \\
\hline All & $1.10(.28)$ & $4.34(1.28)$ & $318(35)$ & $.30(.12)$ & $.37(.32)$ & $.11(.06)$ & \multirow[t]{3}{*}{$.71(.20)$} \\
\hline None & $1.00(.24)$ & 4.37 (1.29) & $274(20)$ & $.28(.10)$ & $.33(.25)$ & $.12(.06)$ & \\
\hline Low/high & $1.16(.28)$ & $4.43(1.06)$ & $301(33)$ & $.38(.13)$ & $.18(.19)$ & $.13(.08)$ & \\
\hline \multicolumn{8}{|l|}{ Experiment 5} \\
\hline $70 \%$ & $1.15(14)$ & $2.98(.62)$ & $412(110)$ & $.37(.08)$ & $.37(.21)$ & $.19(.11)$ & \multirow[t]{3}{*}{$.70(.24)$} \\
\hline $30 \%$ & $1.33(.21)$ & $3.56(.72)$ & $296(43)$ & $.43(.18)$ & $.22(.30)$ & $.17(.08)$ & \\
\hline $0 \%$ & $1.05(.12)$ & $4.14(1.01)$ & $288(.02)$ & $.29(.09)$ & $.43(.51)$ & $.13(.04)$ & \\
\hline
\end{tabular}

Note. The diffusion model assumes that there is intertrial variability in starting point $(s z)$, growth rate $(s v)$, and nondecision time $(s t 0)$. We estimated these variability parameters but did not focus on them because our hypotheses do not make specific predictions about intertrial variability. $a=$ boundary separation; $v=$ growth rate; $t 0=$ nondecision time; $p=$ probability of Kolmogorov-Smirnov statistic used to optimize the fit (larger values indicate better fits).

Diffusion-model fits. Diffusion parameters were estimated for each subject and each run length. Effects of context on boundary separation, growth rate and nondecision time were analyzed by means of separate repeated measures ANOVAs with context and run length as within-subjects factors. The mean values for each parameter appear in Table 5, the estimated decision time and nondecision time are depicted in Figure 5B, and analyses appear in Table 6.

Table 6

Summary of Analyses of Variance for Estimated Diffusion Parameters in Experiments 1-5 (dfs in Parentheses)

\begin{tabular}{|c|c|c|c|c|c|c|}
\hline \multirow[b]{2}{*}{ Experiment and factor } & \multicolumn{2}{|c|}{$a$} & \multicolumn{2}{|c|}{$v$} & \multicolumn{2}{|c|}{ to } \\
\hline & $F$ & $\eta_{p}^{2}$ & $F$ & $\eta_{p}^{2}$ & $F$ & $\eta_{p}^{2}$ \\
\hline Experiment 1 : context $(1,17)$ & $24.5^{* * *}$ & .59 & $6.3^{*}$ & .27 & 8.2 & .33 \\
\hline Experiment 2 : context $(1,17)$ & 0.0 & .00 & $16.5^{* *}$ & .24 & $54.2^{* * *}$ & .51 \\
\hline \multicolumn{7}{|l|}{ Experiment 1 vs. Experiment 2} \\
\hline Context $(1,34)$ & $15.9^{* * *}$ & .32 & 0.5 & .01 & $15.7^{* * *}$ & .32 \\
\hline Experiment $(1,34)$ & $19.0^{* * *}$ & .36 & $5.8^{*}$ & .15 & 1.0 & .03 \\
\hline Context $\times$ Experiment $(1,34)$ & $15.9^{* * *}$ & .32 & $11.4^{* * *}$ & .25 & 2.3 & .14 \\
\hline \multicolumn{7}{|l|}{ Experiment 3} \\
\hline Context $(1,17)$ & $17.2^{* * *}$ & .50 & 2.1 & .11 & $15.8^{* * *}$ & .48 \\
\hline Run length $(2,34)$ & 2.0 & .11 & 0.2 & .01 & 0.8 & .04 \\
\hline Context $\times$ Run Length $(2,34)$ & 1.0 & .06 & 0.1 & .01 & 0.1 & .00 \\
\hline Experiment 4 : context $(2,34)$ & $11.0^{* * *}$ & 39 & 0.4 & .00 & $13.2^{* *}$ & .44 \\
\hline PC: none vs. all $(1,34)$ & $8.1^{* * *}$ & .19 & & & $26.2^{* * *}$ & .43 \\
\hline PC: none vs. low/high $(1,34)$ & $21.7^{* * *}$ & .51 & & & $9.6^{* * *}$ & .22 \\
\hline PC: all vs. low/high $(1,34)$ & 3.2 & .08 & & & $4.0^{\ddagger}$ & .11 \\
\hline Experiment 5 : context $(2,34)$ & $21.5^{* *}$ & .56 & $16.9^{* *}$ & .50 & $22.8^{* * *}$ & .57 \\
\hline PC: $0 \%$ vs. $30 \%(1,34)$ & $41.7^{* * *}$ & .55 & $8.4^{* *}$ & .20 & 0.2 & .00 \\
\hline PC: $0 \%$ vs. $70 \%(1,34)$ & $5.1^{*}$ & .13 & $33.9^{* * *}$ & .50 & $36.4^{* * *}$ & .52 \\
\hline PC: $30 \%$ vs. $70 \%(1,34)$ & $17.6^{* *}$ & .34 & $8.6^{* *}$ & .20 & $31.8^{* * *}$ & .49 \\
\hline
\end{tabular}

Note. When the main effect was significant, we performed planned comparisons (PCs) using the error term of the main effect. $a=$ boundary separation; $v=$ growth rate; $t 0=$ nondecision time

${ }^{*} p<.05 .{ }^{* *} p<.01 .^{\dagger} p=.06 .{ }^{*} p=.05$. 
A

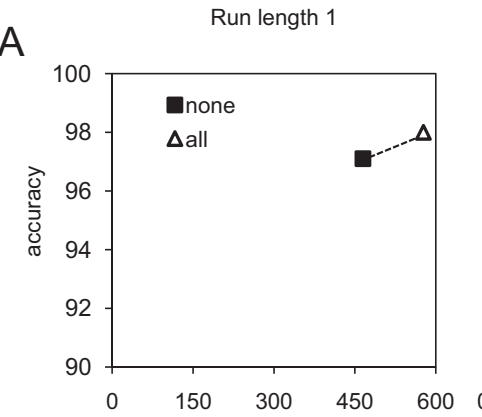

B

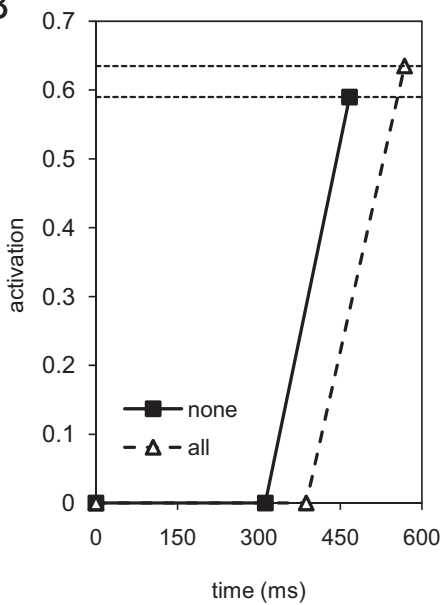

Run length 2

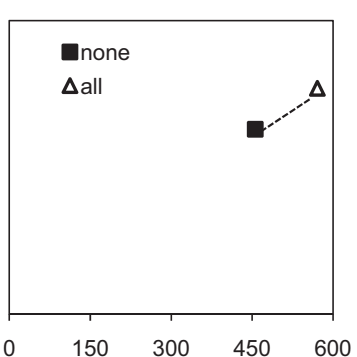

Run length 4

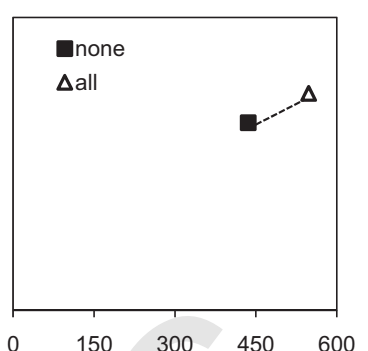

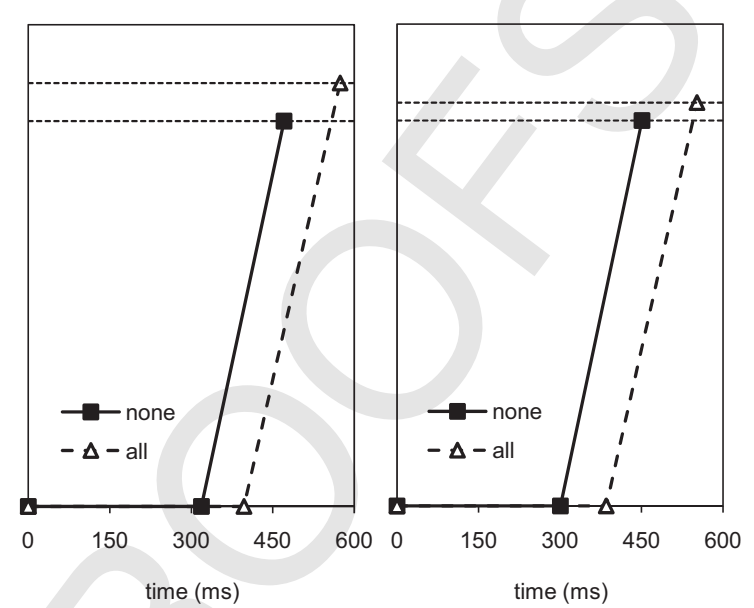

Figure 5. A: Observed reaction times ( $x$-axis) and accuracy ( $y$-axis) for each context and for each run length condition in Experiments 3. B: Estimated decision and nondecision time for each context and for each run length condition in Experiments 3. Growth rate = accumulated evidence per second and response threshold (dotted lines $)=$ boundary separation/2. Decision time $=$ response threshold/growth rate. Nondecision time $=$ stimulus processing + response execution. For purposes of clarity, we depict total nondecision time as the time before the information starts to accumulate.

Replicating the results of Experiment 1, we found significantly greater boundary separation in the all context (1.22) than in the none context (1.14). This effect was not influenced by run length (see Table 6), suggesting that subjects made the same proactive response-strategy adjustments whether a stop signal could occur on the next trial (run length 1 ) or next few trials (run length 2 and run length 4). Stop-signal context increased nondecision time but not growth rate. Run length did not influence these two parameters.

Summary. Consistent with Experiment 1, we found longer RTs and higher accuracy in the all context than in the none context, and these differences were not influenced by run length. The diffusion-model analysis showed that boundary separation was higher in the all context than in the none context, and this difference was also not influenced by run length. Combined, the behavioral data and the diffusion-model fits suggest that subjects make proactive response-strategy adjustments in the stop-signal paradigm, even when the stop-signal context changes on every trial. Thus, the results of Experiment 3 suggest that the discrepancy between our findings and those of Strayer and Kramer (1994) is not due to short run lengths in Strayer and Kramer's experiments.

\section{Experiment 4}

The results of Experiments 1-3 suggest that subjects balance the competing demands of stopping and going by trading speed in the go task for success in the stop task. In Experiment 4, we tested whether these response-strategy adjustments were influenced by the duration of the stop process. We compared a nonselective-stop condition (the all context) with a selectivestop condition, in which subjects had to stop their response when one tone occurred but not when another tone occurred. Riegler (1986) found longer SSRTs in selective stopping than in nonselective stopping, reflecting the greater demands on the stop process. To implement selective stopping, we included two cues in addition to the standard 'all' and 'none' cues: 'low,' which instructed subjects to inhibit responses when a low tone occurred but not when a high tone occurred, and 'high,' which instructed subjects to inhibit responses when a high tone occurred but not when a low tone occurred. We expected longer SSRTs following 'high' and 'low' than following 'all'. If proactive response-strategy adjustments are influenced by the duration of the stop process, then we should observe longer RTs in the low/high context than in the all context because SSRT 
should be longer in the low/high context than in the all context. By contrast, if proactive response-strategy adjustments are influenced by the outcome of the stop process, then we should observe similar RTs in the low/high and all contexts because the procedure that tracked SSD should produce similar values of $\mathrm{p}$ (respondlsignal) in the two contexts.

\section{Method}

Subjects. Eighteen students from Vanderbilt University participated for course credit. All subjects had normal or correctedto-normal vision and all were naive as to the purpose of the experiment.

Apparatus, stimuli, and procedure. These were the same as in Experiment 1 except for the following: Half of the trials were low/high context trials, one-quarter were none context trials, and one-quarter were all context trials. We used these proportions to ensure that at the end of the experiment, we had an equal number of relevant signals in the low/high context and the all context.

In the low/high context, the SSD for relevant stop signals was initially set at $150 \mathrm{~ms}$ and continuously adjusted according to same tracking procedure as in the all context. SSD for irrelevant stop signals was the same as the current SSD for relevant stop signals in the low/high context; note that SSD was not adjusted after irrelevant stop signals.

\section{Results and Discussion}

We used the same exclusion criteria as in Experiment 1. Mean RT for included trials was calculated after exclusions of outliers $(2.2 \%)$.

Signal analyses. For all three contexts, we compared performance on no-stop-signal trials and stop-signal trials first. RT, accuracy, and omission rate are presented in Table 2; a summary of the ANOVAs is presented in Table 3.

RT was longer for stop-signal trials (415 ms) than for no-stopsignal trials $(381 \mathrm{~ms})$ in the none context; the differences in omission rate and accuracy were not significant. Consistent with the previous experiments and the horse-race model, we found that signal-respond RTs (433 ms) were shorter than no-stop-signal RTs (453 ms) in the all context, although this difference was not significant. Accuracy was comparable for no-stop-signal and signal-respond trials. In the low/high context, RTs were longer for invalid stop-signal trials $(599 \mathrm{~ms})$ than for no-stop-signal trials (437 ms) and signal-respond trials (401 ms). These findings replicate previous studies (e.g., De Jong, Coles, \& Logan, 1995), and suggest that making a decision about the validity of the stop signal interfered with responding to the go task. Omission rate was also higher for invalid-stop-signal trials $(6.4 \%)$ than for no-stop-signal trials $(0.6 \%)$, suggesting that subjects incorrectly stopped the response on a proportion of the invalid-stop-signal trials. Go accuracy was comparable for the different trial types in the low/high context.

We also compared stopping performance in relevant stop-signal trials in the low/high context and the all context. P(respondlsignal), SSD, and SSRT are presented in Table 1. Mean p(respondlsignal) was comparable in both contexts $(p>.13)$. In line with Riegler (1986), we found that SSRT was longer in the low/high context
( $259 \mathrm{~ms})$ than in the all context (229 ms), although this difference just failed to reach significance, $F(1,17)=4.2, M S E=1,799, p=$ $.06, \eta_{p}^{2}=.20$.

Context analyses. To determine whether proactive responsestrategy adjustments are affected by the demands of the stop task, we analyzed no-stop-signal RTs by means of a repeated measures ANOVA with context as a within-subjects factor. A summary of the ANOVAs is presented in Table 4. Planned comparisons were performed using the error term of the global analysis. Mean RTs and accuracy scores are depicted in Figure 6A; standard deviations F6 appear in Table 2. Because there were not enough observations for all positions in the none and all context, we did not test the interaction between context and position in a run.

Consistent with the proactive-adjustment hypothesis, there was a main effect of context for both RT data and accuracy data. Planned comparisons demonstrated that RTs were longer in the all (453 ms) and the low/high context $(437 \mathrm{~ms})$ than in the none context (381 ms). The difference between RTs in the low/high and the all context was not significant. Planned comparisons demonstrated that accuracy was higher in the all (98.3\%) and low/high context $(97.8 \%)$ than in the none context $(96.3 \%)$. Accuracy was

A

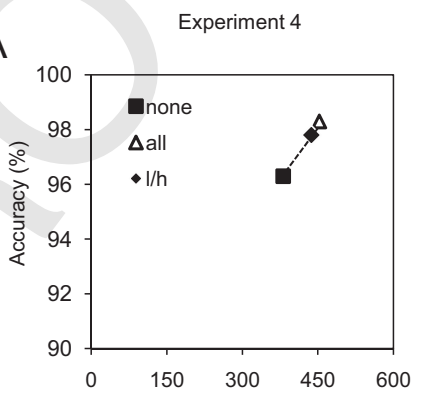

Experiment 5

B
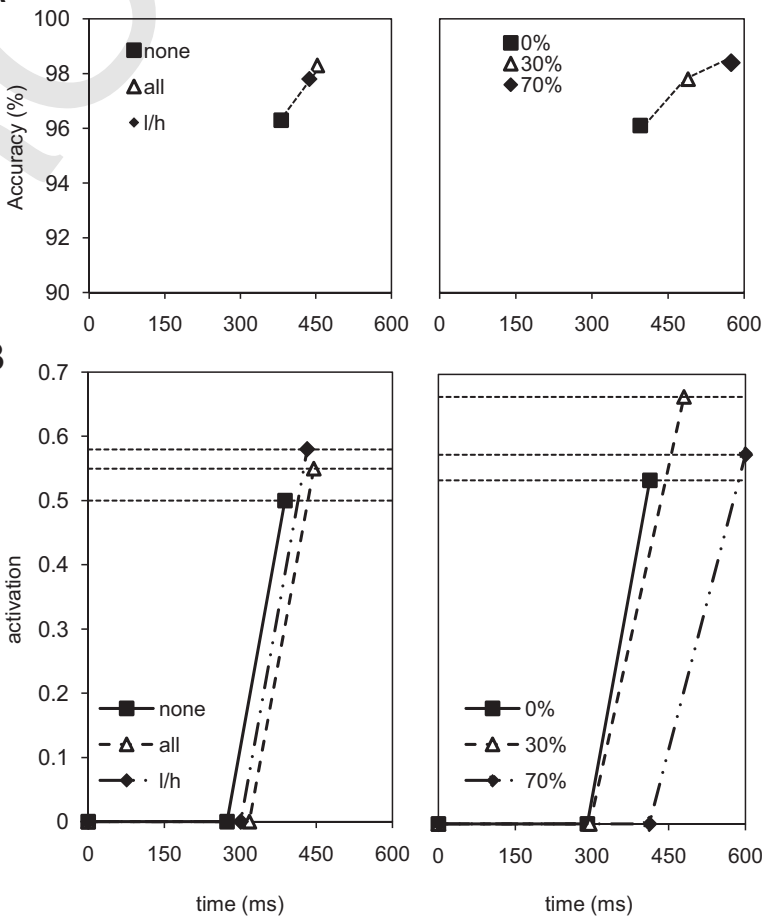

Figure 6. A: Observed RT ( $x$-axis) and accuracy ( $y$-axis) for each context for Experiments 4 and 5. Note: 1/h $=$ low/high context. B: Estimated decision and nondecision time for each context for Experiments 4 and 5. Note: $1 / \mathrm{h}=$ low $/$ high context. Growth rate $=$ accumulated evidence per second and response threshold (dotted lines) = boundary separation/2. Decision time $=$ response threshold/growth rate. Nondecision time $=$ stimulus processing + response execution. For purposes of clarity, we depict total nondecision time as the time before the information starts to accumulate. 
comparable in the 'low/high' and all context. Combined, these findings suggest that the response threshold was increased when subjects expected relevant stop signals to occur, but these proactive adjustments were not influenced by the difficulty of the stop task.

Diffusion-model fits. Diffusion parameters were estimated for each subject and each context condition. Because of the lower number of trials in the all and none contexts (about 60 trials per context per subject), we allowed variability in starting point, nondecision time and growth rate to differ between the conditions. This let us use data from all subjects; without allowing variability to differ between the conditions, model fits were poor for certain subjects (i.e., the predicted distribution differed significantly from the observed distribution). Effects of context on boundary separation, growth rate, and nondecision time were analyzed by means of separate repeated measures ANOVAs with context as a within-subjects factor. The mean values for each parameter appear in Table 5 and the estimated decision time and nondecision time are depicted in Figure 6B; analyses appear in Table 6.

Replicating the results of Experiment 1, we found that boundary separation was influenced by the context. Planned comparisons showed that boundary separation was higher in the all (1.10) and low/high (1.16) context than in the none context (1.00). The difference between the all and low/high context was not significant. These findings are consistent with the behavioral results and suggest that subjects made similar responsestrategy adjustments in the low/high and all contexts. Thus, proactive response-strategy adjustments seem to depend more on the outcome than on the duration of the stop process. Nondecision time was also influenced by stop-signal context. Planned comparisons showed that nondecision time was longer in the all $(318 \mathrm{~ms})$ and low/high context $(301 \mathrm{~ms})$ than in the none context $(274 \mathrm{~ms})$. The difference between the all and low/high context failed to reach significance. Growth rate was not influenced by stop-signal context.

Summary. In Experiment 4, we asked whether the duration of the stop process influenced proactive response-strategy adjustments. Replicating previous studies, we found that SSRT was longer in the low/high context than in the all context. However, RTs, accuracy, and boundary separation were comparable in the low/high context and the all context. These findings suggest that proactive response-strategy adjustments depend more on the outcome of the stop process, that is, $\mathrm{p}$ (respondlsignal), than on the duration of the stop process (i.e., SSRT).

\section{Experiment 5}

In Experiment 5, we examined whether proactive responsestrategy adjustments are influenced by the proportion of stop signals. Logan (1981; Logan \& Burkell, 1986) found that RTs increased when the proportion of stop signals increased. To determine whether these differences could be produced proactively on a trial-by-trial basis, we used three context cues: $0 \%$, $30 \%$, and $70 \%$, each indicating the probability that a stop signal would be presented on the next trial. Unlike the none context of the other experiments, no stop signals were presented in the $0 \%$ context.

\section{Method}

Subjects. Eighteen students from Vanderbilt University participated for course credit. All subjects had normal or correctedto-normal vision and all were naive as to the purpose of the experiment.

Apparatus, stimuli, and procedure. The go task was the same as in Experiment 1. We used three context cues, each indicating the probability that a stop signal would be presented: $0 \%(11 \times$ $6 \mathrm{~mm}), 30 \%(16 \times 6 \mathrm{~mm})$, or $70 \%(16 \times 6 \mathrm{~mm})$. Thus, stop signals were presented only in the $30 \%$ and $70 \%$ contexts. Trials were divided in runs of 4 or 6 trials in the same context. Run length was randomized with the same restrictions used in Experiment 1. One-third of the trials were $0 \%$ context trials, one-third of the trials were $30 \%$ context trials, and one-third of the trials were $70 \%$ context trials. The experiment started with a short practice block of 24 trials, followed by 14 experimental blocks of 60 trials.

\section{Results and Discussion}

The exclusion criteria were the same as in Experiment 1. Mean RT for included trials was calculated after removal of outlying RTs $(3.2 \%)$.

Signal analyses. $\mathrm{P}($ respondlsignal), mean SSD, and mean SSRT are presented in Table 1. As can be seen, $\mathrm{p}$ (respondlsignal) was comparable for the $30 \%$ context and the $70 \%$ context $(F<1)$. We found that SSRT was similar in the 30\% (226 ms) and 70\% context $(213 \mathrm{~ms}, F<1)$. This finding suggests that stopping performance was not influenced by stop-signal probability (Logan \& Burkell, 1986) or by proactive response-strategy adjustments (see below).

RT, accuracy and omission rates are presented in Table 2. In the $30 \%$ and $70 \%$ context, signal-respond RTs (424 ms and $513 \mathrm{~ms}$, respectively) were shorter than no-stop-signal RTs (489 ms and $574 \mathrm{~ms}$, respectively). In both contexts, accuracy was comparable for signal trials and no-stop-signal trials.

Context analyses. To examine whether stop-signal probability influenced proactive response-strategy adjustments, we analyzed no-stop-signal performance by means of a repeated measures ANOVA with context $(0 \%, 30 \%$, or $70 \%)$ as a within-subjects factor. A summary of the ANOVAs is presented in Table 4. Observed RTs and accuracy scores are depicted in Figure 6A; standard deviations appear in Table 2. For the $0 \%$ and 30\% contexts, we tested the interaction between context and position; these analyses appear in Appendix C.

Overall, we found a main effect of context for no-stop-signal RT, accuracy, and omission rate. Planned comparisons showed that nostop-signal RT was longer in the $30 \%$ context (489 ms) and $70 \%$ context $(574 \mathrm{~ms})$ than in the $0 \%$ context $(395 \mathrm{~ms})$ and was longer in the $70 \%$ context than in the $30 \%$ context. Planned comparisons showed that accuracy was higher in the $30 \%$ context $(97.8 \%)$ and $70 \%$ contexts $(98.4 \%)$ than in the $0 \%$ context $(96.1 \%)$, but the difference between the $30 \%$ and the $70 \%$ contexts was not significant. Combined, the RT and accuracy data suggest that subjects made proactive response-strategy adjustments when relevant stop signals could occur. The RT difference between the $30 \%$ and $70 \%$ contexts suggests that subjects made greater adjustments when stopsignal probability was high. 
Diffusion-model fits. Diffusion parameters were estimated for each subject and each context condition. As in Experiment 4 , we allowed variability in starting point, nondecision time, and growth rate to differ between the conditions. Effects of context on boundary separation, growth rate, and nondecision time were analyzed by means of separate repeated measures ANOVAs with context as a within-subjects factor. The mean values for each parameter appear in Table 5, and the estimated decision time and nondecision time are depicted in Figure 6B; analyses appear in Table 6.

Replicating the previous experiments, we found that boundary separation was influenced by the stop-signal context: Planned comparisons showed that boundary separation was greater in the $30 \%$ context (1.33) than in the $0 \%$ context (1.05). Planned comparisons found no significant difference in nondecision time between the $0 \%$ context $(287 \mathrm{~ms})$ and the $30 \%$ context (296 ms). This small numerical difference could be due to dual-task requirements. Note that we did observe significant and large nondecision differences in Experiments 1 and 3-4, which may have been influenced by response-strategy adjustments. We will discuss this issue further in the General Discussion. Consistent with Experiment 1 (but inconsistent with Experiments 3 and 4), we found a significantly higher growth rate in the $0 \%$ context $(4.14$ units $/ \mathrm{sec})$ than in the $30 \%$ context (3.6 units/sec). There were also differences between the $70 \%$ context and the $0 \%$ and $30 \%$ contexts (see Tables 5 and 6). However, the results for the $70 \%$ context should be interpreted with caution because of the very low number of trials (about 21 trials per subject), which may not be enough to get reliable parameter estimates (Voss \& Voss, 2007). ${ }^{3}$

Summary. We found that RTs were longer and accuracy was higher in the $30 \%$ and $70 \%$ contexts than in the $0 \%$ context, suggesting proactive response-strategy adjustments. Furthermore, we found that RTs were longer in the $70 \%$ context than in the $30 \%$ context, suggesting that subjects made greater adjustments when the stop-signal probability was higher. This finding is consistent with previously observed differences between blocks with low and high proportions of stop signals (Logan, 1981; Logan \& Burkell, 1986) and suggests that proactive response-strategy adjustments within a block are similar to proactive response-strategy adjustments at the beginning of a block.

\section{General Discussion}

In the present study, we examined whether subjects can proactively change response strategies in the stop-signal paradigm by presenting precues that informed subjects about the occurrence of stop signals on the next few trials. We tested two hypotheses: the proactive-adjustment hypothesis and the dualtask requirements hypotheses. Over all experiments, the results were consistent with both hypotheses: In the stop-signal experiments (Experiments 1, 3-5), RT and go accuracy were higher in stop-signal contexts than in no-stop-signal contexts, suggesting that subjects increased the response threshold in the stopsignal contexts, even when the context changed after every trial (Experiment 3). This conclusion was supported by the diffusion-model fits, which showed that response threshold was higher in stop-signal contexts than in no-stop-signal contexts. These effects are consistent with the proactive-adjustment hy- pothesis. However, Experiment 2 showed that RT but not accuracy was influenced by dual-task requirements, and the diffusion-model fits showed that nondecision time but not response threshold was influenced by dual-task requirements. This nondecision time effect in the dual-task experiment suggests that the nondecision time effects in the stop-signal experiments may be (partly) due to dual-task requirements. Growthrate differences were inconsistent across the experiments. Growth rate was higher in the dual-task condition than in the single-task condition of Experiment 2, which is paradoxical. It was lower in the stop-signal context than in the no-stop-signal context in Experiments 1 and 5, but it did not differ significantly in Experiments 3 and 4. Therefore, the behavioral data and the diffusion-model fits suggest that dual-task requirements mainly influenced nondecision processes, increasing RTs in stop-signal and dual-task contexts.

In the remainder of the article, we further discuss the proactive response-strategy adjustment and dual-task requirements hypotheses, and we consider two alternative hypotheses: the delayedprocessing hypothesis and the response-suppression hypothesis. We conclude with some practical guidelines for stop-signal paradigm users.

\section{The Role of Proactive Response-Strategy Adjustment in Cognitive Control}

The findings of the present study demonstrate that subjects can make proactive response-strategy adjustments on a trial-bytrial basis, suggesting a flexible cognitive control system that can adjust itself to achieve a balance between competing goals. Response-strategy adjustments are typically investigated in choice RT tasks in which subjects balance speed and accuracy. We think that there may be important parallels between adjustments in choice RT tasks and adjustments in the stop-signal task. Several choice RT studies showed that subjects trade speed for accuracy after errors or after conflict is detected (e.g., Botvinick et al., 2001; Burns, 1971; Holroyd, Yeung, Coles, \& Cohen, 2005; Rabbitt, 1966); similarly, stop-signal studies showed that subjects trade speed in the go task for success in the stop task after stop-signal trials (e.g., Rieger \& Gauggel, 1999; Verbruggen \& Logan, in press; Verbruggen, Logan, Liefooghe, et al., 2008). Several studies demonstrated that subjects trade speed for accuracy in choice RT tasks when instructions vary between blocks (e.g., Howell \& Kreidler, 1963; Pachella, 1974; Rinkenauer et al., 2004); similarly, subjects trade speed in the go task for success in stopping in stop-signal blocks (e.g., Logan, 1981; Stuphorn \& Schall, 2006; Verbruggen et al., 2004). Combined, these studies show that subjects can make proactive response-strategy adjustments at the beginning of a block and then make trial-by-trial reactive adjustments during the block. Whether subjects can also make proactive response-strategy adjustments on a trial-by-trial basis in choice RT tasks is still debated. Several studies showed that subjects did not adjust response strategies proactively when

\footnotetext{
${ }^{3}$ For all parameters, we found similar differences between the $0 \%$ context and the $30 \%$ context when we excluded $70 \%$ context trials from the diffusion-model fits.
} 
precues informed them about upcoming item types (Brown \& Steyvers, 2005; Los, 1999; Strayer \& Kramer, 1994). Other studies observed proactive response-strategy adjustments but only when the precues explicitly instruct subjects to respond as quickly as possible (Gopher, Armony, \& Greenshpan, 2000; Kleinsorge, 2001; Los, 1999) or when the use of iteminformative precues was strongly emphasized (Logan \& Zbrodoff, 1982; for similar ideas about the use of precues, see Verbruggen et al. 2007). In the present study, precues informed subjects about the occurrence of stop signals on the next few trials. Therefore, one of the major contributions of the present study is to show that subjects are capable of adjusting decision criteria proactively on a trial-by-trial basis, even without the explicit instruction to adjust response strategies.

The similarities between these response-strategy adjustments in choice RT and stopping suggest subjects make the same adjustments in the different paradigms. One way of interpreting the similarities is that subjects manipulate the speed-accuracy tradeoff in both paradigms. Indeed, trading speed for accuracy in the go task increases success rate in the stop task (Logan, 1981). The results of the present study are consistent with this idea: When subjects expect stop signals to occur, both RTs and go accuracy increase. The diffusion model (e.g., Ratcliff, 2006; Ratcliff et al., 1999; Usher \& McClelland, 2001) provides new insight into this tradeoff: Response-strategy adjustments are implemented by manipulating response threshold (boundary separation). We assume that the similarities exist not because subjects intentionally trade speed for accuracy in the two paradigms but because they intentionally adjust response threshold to control speed and that changes in go accuracy in the stop-signal paradigm are an unintended consequence of this adjustment.

More generally, we assume that response-threshold adjustments in choice RT and stop-signal tasks are an integral part of cognitive control (Logan, 1985). Many theorists argue that executive processes exert control by manipulating the goal representations in response to changes in internal states or changes in the environment (Logan \& Cowan, 1984; Logan \& Gordon, 2001; Meyer \& Kieras, 1997; Miller \& Cohen, 2001). We elaborate this idea by suggesting that executive processes also adjust response strategies to achieve a balance between opposing goals in response to conflicting or ambiguous instructions (see also e.g., Howell \& Kreidler, 1963; Logan, 1985; Logan \& Gordon, 2001; Logan \& Zbrodoff, 1982).

\section{Dual-Task Costs in the Stop-Signal Paradigm}

The results of the present study showed that the increased RTs in stop-signal contexts are due to dual-task requirements as well as proactive response-strategy adjustments. The dual-task cost seems inconsistent with previous studies that showed that stopping is hardly influenced by go processing (Logan \& Burkell, 1986; Logan \& Cowan, 1984; Logan et al., 1984; Verbruggen, Schneider, et al., in press). For example, Logan and Cowan (1984) demonstrated that SSRT did not show the typical dual-task interference effect (or psychological-refractoryperiod effect), which is commonly observed when subjects have to execute two responses in rapid succession (see Pashler, 1994). However, the absence of a psychological-refractoryperiod effect on SSRT does not preclude the presence of dual- task concurrence costs in the go task. Indeed, Logan et al. (1984) found no refractory-period effect, but they found a general slowing in stopping performance in a choice RT task compared to a simple RT task and suggested that the SSRT difference was due to resource competition between stop processes and go processes. Similarly, Logan and Burkell (1986) suggested that there is a concurrence cost in advance task preparation when subjects are preparing to respond to different stimuli. These ideas are consistent with the results of the present study and are in line with the dual-task requirement hypothesis, which assumes a general (but relatively small) concurrence cost in the stop-signal paradigm.

\section{The Delayed-Responding Hypothesis and the Response- Suppression Hypothesis}

The results of Experiment 2 suggested that dual-task requirements influenced nondecision time. However, as can be seen in Table 5 and in Figures 4-6, the nondecision time differences in Experiments 1 and 3-4 (see also the 70\% context of Experiment 5) were generally larger than the nondecision time difference in Experiment 2. Because there are no a priori reasons to assume that dual-task requirements should influence nondecision time processes differently in stop-signal and dual-task contexts, the diffusion-model fits suggest that the nondecision time differences in the stop-signal experiments were not solely due to dual-task requirements. We consider two hypotheses that might account for the larger nondecision time differences in the stop-signal experiments: the delayed-processing hypothesis and the responsesuppression hypothesis.

The delayed-processing hypothesis assumes that subjects postpone processing of the go stimulus, the execution of the go response, or both, until they know they can safely execute the response. This would affect the nondecision time parameter in the diffusion model fits. However, delayed processing is implausible for several reasons. First, SSD changes after every stop-signal trial, making it difficult to estimate the next SSD accurately. Second, subjects also need to estimate SSRT to determine whether they have enough time to inhibit the response at a given SSD. Estimating SSRT may be difficult because there is no overt response to the stop signal. Third, time estimation is an effortful process, and previous studies showed strong dual-task interference effects when subjects estimated time intervals while performing a concurrent task (see, e.g., Brown, 2006; Vandierendonck, De Vooght, \& Van der Goten, 1998a). Dual-task interference would make time estimation in the stop-signal paradigm even more difficult than it already is. Therefore, we conclude that the delayed-responding hypothesis is implausible.

The response-suppression hypothesis assumes that opposing task goals suppress motor output and this effect is particularly strong in the stop-signal paradigm. Frank (2006) proposed a computational model of the basal ganglia circuitry and suggested that temporary suppression of the motor system slows down responding in cognitively demanding situations: Fast responses are prevented through activation of the subthalamic nucleus, which excites the internal segment of the globus pallidus, making the thalamus more inhibited. This suppression would appear in nondecision time in diffusion-model fits. Based on Frank's model, the responsesuppression hypothesis assumes that subjects may temporarily 
suppress all motor output when they expect stop signals on the next trial or trials (see also, e.g., Aron, Behrens, Smith, Frank, \& Poldrack, 2007; Stuphorn \& Schall, 2006; van den Wildenberg, van der Molen, \& Logan, 2002). This does not imply that subjects will not increase the response threshold in stop-signal blocks; these control adjustments are not mutually exclusive so subjects may do both (see also, Rinkenauer et al., 2004, who showed that speedaccuracy instructions influenced both decisional and nondecisional processes).

A final remark concerns the growth-rate differences between the none and all contexts in Experiments 1 and 5. Experiment 2 showed that growth rate does not decrease in dual-task contexts. This suggests that lower growth rates in the stop-signal contexts of Experiments 1 and 5 were not due to dual-task requirements. It is unclear how response strategies could influence growth rate of information. One possibility is that all ongoing processing (including accumulation of information) is suppressed in stop-signal contexts. To our knowledge, strategic effects on growth rate have not been studied systematically, so further research is needed here.

\section{Practical Guidelines}

The results of the present study suggest that subjects change response thresholds and suppress motor output when they expect stop signals to occur. This finding has some practical implications for researchers using the stop-signal paradigm. Researchers usually tell subjects not to wait for a stop signal, but subjects slow their RTs nevertheless. We showed that the slowing is greater when the proportion of stop signals increases. Therefore, it is advisable to keep the proportion of stop signals as low as possible.

Because of response-strategy adjustments, it is also advisable to use the tracking procedure to adjust SSD. The tracking procedure adjusts SSD to account for differences in RT differences, keeping $\mathrm{p}$ (respondlsignal) close to .50 , which produces the most reliable SSRT estimates (Band, van der Molen, \& Logan, 2003). By contrast, the fixed-SSD procedure does not account for differences in $\mathrm{RT}$, so $\mathrm{p}$ (respondlsignal) may be influenced by proactive response-strategy adjustments (see Figure 2A vs. Figure 2D) and produce less reliable SSRT estimates (see Figure 2D). Therefore, we advise researchers to use the tracking procedure in combination with a low proportion of stop-signal trials.

When comparing stopping performance between groups, researchers sometimes compare RTs to determine whether the observed SSRT differences are due to differences in inhibitory processes or to general processes that influence both executing and stopping a response (see, e.g., Ridderinkhof et al., 1999). Our results suggest that subjects typically make response-strategy adjustments when they expect stop signals to occur, so increased RTs may reflect adjusted control settings instead of impaired performance on the go task. Therefore, we advise researchers to include a no-stop-signal control block when they want to examine both go-task and stop-task differences (see, e.g., Verbruggen et al., 2005, 2004).

\section{Conclusions}

In the present study, we demonstrated that subjects can adjust response strategies proactively to achieve a balance between competing task goals on a trial-by-trial basis. We found longer RT and higher accuracy when precues indicated that stop signals were likely, which we attributed primarily to response-strategy adjustments. Dual-task requirements played a smaller role. Diffusionmodel fits showed that these response-strategy adjustments involved increasing the response threshold and, possibly, suppressing the motor output, which is consistent with the idea that control adjustments can influence both decisional and nondecisional stages (see, e.g., Frank, 2006; Rinkenauer et al., 2004). An important goal for future research is to explicate the relations between these proactive response-strategy adjustments and reactive response strategy adjustments seen in other studies.

\section{References}

Aron, A. R., Behrens, T. E., Smith, S., Frank, M. J., \& Poldrack, R. A. (2007). Triangulating a cognitive control network using diffusionweighted magnetic resonance imaging (MRI) and functional MRI. Journal of Neuroscience, 27, 3743-3752.

Baddeley, A. (1996). Exploring the central executive. Quarterly Journal of Experimental Psychology, 49A, 5-28.

Band, G. P. H., van der Molen, M. W., \& Logan, G. D. (2003). Horse-race model simulations of the stop-signal procedure. Acta Psychologica, 112, 105-142.

Bedard, A. C., Nichols, S., Barbosa, J. A., Schachar, R., Logan, G. D., \& Tannock, R. (2002). The development of selective inhibitory control across the life span. Developmental Neuropsychology, 21, 93-111.

Botvinick, M. M., Braver, T. S., Barch, D. M., Carter, C. S., \& Cohen, J. D. (2001). Conflict monitoring and cognitive control. Psychological Review, 108, 624-652.

Brown, S., \& Steyvers, M. (2005). The dynamics of experimentally induced criterion shifts. Journal of Experimental Psychology: Learning, Memory, and Cognition, 31, 587-599.

Brown, S. W. (2006). Timing and executive function: Bidirectional interference between concurrent temporal production and randomization tasks. Memory \& Cognition, 34, 1464-1471.

Burns, J. T. (1971). Error-induced inhibition in a serial reaction time task. Journal of Experimental Psychology, 90, 141-148.

De Jong, R., Coles, M. G. H., \& Logan, G. D. (1995). Strategies and mechanisms in nonselective and selective inhibitory motor control. Journal of Experimental Psychology: Human Perception and Performance, 21, 498-511.

Emeric, E. E., Brown, J. W., Boucher, L., Carpenter, R. H. S., Hanes, D. P., Harris, R., et al. (2007). Influence of history on saccade countermanding performance in humans and macaque monkeys. Vision Research, 47, 35-49.

Frank, M. J. (2006). Hold your horses: A dynamic computational role for the subthalamic nucleus in decision making. Neural Networks, 19, 1120-1136.

Gopher, D., Armony, L., \& Greenshpan, Y. (2000). Switching tasks and attention policies. Journal of Experimental Psychology: General, 129, 308-339.

Gratton, G., Coles, M. G. H., \& Donchin, E. (1992). Optimizing the use of information: Strategic control of activation of responses. Journal of Experimental Psychology: General, 121, 480-506.

Holroyd, C. B., Yeung, N., Coles, M. G. H., \& Cohen, J. D. (2005). A mechanism for error detection in speeded response time tasks. Journal of Experimental Psychology: General, 134, 163-191.

Howell, W. C., \& Kreidler, D. L. (1963). Information processing under contradictory instructional sets. Journal of Experimental Psychology, 65, 39-46.

Kleinsorge, T. (2001). The time course of effort mobilization and strategic adjustments of response criteria. Psychological Research, 65, 216-233.

Lappin, J. S., \& Eriksen, C. W. (1966). Use of a delayed signal to stop a 
visual reaction-time response. Journal of Experimental Psychology, 72, $805-811$.

Logan, G. D. (1981). Attention, automaticity, and the ability to stop a speeded choice response. In J. Long and A. D. Baddeley (Eds.), Attention and Performance IX (pp. 205-222). Hillsdale, NY: Erlbaum.

Logan, G. D. (1985). Executive control of thought and action. Acta Psychologica, 60, 193-210.

Logan, G. D., \& Bundesen, C. (2003). Clever homunculus: Is there an endogenous act of control in the explicit task-cuing procedure? Journal of Experimental Psychology: Human Perception and Performance, 29, 575-599.

Logan, G. D., \& Burkell, J. (1986). Dependence and independence in responding to double stimulation: A comparison of stop, change, and dual-task paradigms. Journal of Experimental Psychology: Human Perception and Performance, 12, 549-563.

Logan, G. D., \& Cowan, W. B. (1984). On the ability to inhibit thought and action: A theory of an act of control. Psychological Review, 91, 295-327.

Logan, G. D., Cowan, W. B., \& Davis, K. A. (1984). On the ability to inhibit simple and choice reaction-time responses: A model and a method. Journal of Experimental Psychology: Human Perception and Performance, 10, 276-291.

Logan, G. D., \& Gordon, R. D. (2001). Executive control of visual attention in dual-task situations. Psychological Review, 108, 393-434.

Logan, G. D., Schachar, R. J., \& Tannock, R. (1997). Impulsivity and inhibitory control. Psychological Science, 8, 60-64.

Logan, G. D., \& Zbrodoff, N. J. (1979). When it helps to be misled: Facilitative effects of increasing the frequency of conflicting stimuli in a Stroop-like task. Memory \& Cognition, 7, 166-174.

Logan, G. D., \& Zbrodoff, N. J. (1982). Constraints on strategy construction in a speeded discrimination task. Journal of Experimental Psychology: Human Perception and Performance, 8, 502-520.

Los, S. A. (1999). Identifying stimuli of different perceptual categories in pure and mixed blocks of trials: Evidence for stimulus-driven switch costs. Acta Psychologica, 103, 173-205.

Meyer, D. E., \& Kieras, D. E. (1997). A computational theory of executive cognitive processes and multiple-task performance. I. Basic mechanisms. Psychological Review, 104, 3-65.

Miller, E. K., \& Cohen, J. D. (2001). An integrative theory of prefrontal cortex function. Annual Review of Neuroscience, 24, 167-202.

Miyake, A., Friedman, N. P., Emerson, M. J., Witzki, A. H., Howerter, A., \& Wager, T. D. (2000). The unity and diversity of executive functions and their contributions to complex "frontal lobe" tasks: A latent variable analysis. Cognitive Psychology, 41, 49-100.

Pachella, R. G. (1974). The interpretation of reaction time in information processing research. In B. Kantowitz (Ed.), Human information processing: Tutorials in performance and cognition (pp. 41-82). New York: Halstead Press.

Pashler, H. (1994). Dual-task interference in simple tasks: Data and theory. Psychological Bulletin, 116, 220-244.

Rabbitt, P. M. A. (1966). Errors and error correction in choice-response tasks. Journal of Experimental Psychology, 71, 264-272.

Rabbitt, P. M. A. (1968). Repetition effects and signal classification strategies in serial choice-response tasks. Quarterly Journal of Experimental Psychology 20, 232-240.

Ratcliff, R. (1978). A theory of memory retrieval. Psychological Review, 85, 59-108.

Ratcliff, R. (2006). Modeling response signal and response time data. Cognitive Psychology, 53, 195-237.

Ratcliff, R., Van Zandt, T., \& McKoon, G. (1999). Connectionist and diffusion models of reaction time. Psychological Review, 106, 261-300.

Ridderinkhof, K. R., Band, G. P. H., \& Logan, G. D. (1999). A study of adaptive behavior: Effects of age and irrelevant information on the ability to inhibit one's actions. Acta Psychologica, 101, 315-337.
Rieger, M., \& Gauggel, S. (1999). Inhibitory after-effects in the stop signal paradigm. British Journal of Psychology, 90, 509-518.

Riegler, G. L. (1986). Are stop and go processes independent in the stop-signal paradigm? Unpublished master's thesis, Purdue University, West Lafayette, IN.

Rinkenauer, G., Osman, A., Ulrich, R., Muller-Gethmann, H., \& Mattes, S. (2004). The locus of speed-accuracy trade-off in reaction time: Inferences from the lateralized readiness potential. Journal of Experimental Psychology: General, 133, 261-282.

Schachar, R. J., Chen, S., Logan, G. D., Ornstein, T. J., Crosbie, J., Ickowicz, A., \& Pakulak, A. (2004). Evidence for an error monitoring deficit in attention deficit hyperactivity disorder. Journal of Abnormal Child Psychology, 32, 285-293.

Spence, C., \& Driver, J. (1997). Cross-modal links in attention between audition, vision and touch: Implications for interface design. International Journal of Cognitive Ergonomics, 1, 351-373.

Stevens, M., Lammertyn, J., Verbruggen, F., \& Vandierendonck, A. (2006). Tscope: A C library for programming cognitive experiments on the MS Windows platform. Behavior Research Methods, 38, 280-286.

Strayer, D. L., \& Kramer, A. F. (1994). Strategies and automaticity: II. Dynamic aspects of strategy adjustment. Journal of Experimental Psychology: Learning, Memory, and Cognition, 20, 342-365.

Stuphorn, V., \& Schall, J. D. (2006). Executive control of countermanding saccades by the supplementary eye field. Nature Neuroscience, 9, 925-931.

Usher, M., \& McClelland, J. L. (2001). The time course of perceptual choice: The leaky, competing accumulator model. Psychological Review, 108, 550-592.

van Boxtel, G. J. M., van der Molen, M. W., \& Jennings, J. R. (2005). Differential involvement of the anterior cingulate cortex in performance monitoring during a stop-signal task. Journal of Psychophysiology, 19, $1-10$

van den Wildenberg, W. P. M., van der Molen, M. W., \& Logan, G. D. (2002). Reduced response readiness delays stop signal inhibition. Acta Psychologica, 111, 155-169.

Vandierendonck, A., De Vooght, G., \& Van der Goten, K. (1998a). Does random time interval generation interfere with working memory executive functions? European Journal of Cognitive Psychology, 10, 413-442.

Vandierendonck, A., De Vooght, G., \& Van der Goten, K. (1998b). Interfering with the central executive by means of a random interval repetition task. Quarterly Journal of Experimental Psychology Section Human Experimental Psychology, 51(A), 197-218.

Verbruggen, F., Liefooghe, B., Notebaert, W., \& Vandierendonck, A. (2005). Effects of stimulus-stimulus compatibility and stimulusresponse compatibility on response inhibition. Acta Psychologica, 120, 307-326.

Verbruggen, F., Liefooghe, B., \& Vandierendonck, A. (2004). The interaction between stop signal inhibition and distractor interference in the flanker and Stroop task. Acta Psychologica, 116, 21-37.

Verbruggen, F., Liefooghe, B., \& Vandierendonck, A. (2006). The effect of interference in the early processing stages on response inhibition in the stop signal task. Quarterly Journal of Experimental Psychology, 59, 190-203.

Verbruggen, F., Liefooghe, B., Vandierendonck, A., \& Demanet, J. (2007). Short cue presentations encourage advance task preparation: A recipe to diminish the residual switch cost. Journal of Experimental Psychology: Learning, Memory, and Cognition, 33, 342-356.

Verbruggen, F., \& Logan, G. D. (in press). Long-term aftereffects of response inhibition: Memory retrieval, task goals, and cognitive control. Journal of Experimental Psychology: Human Perception and Performance.

Verbruggen, F., Logan, G. D., Liefooghe, B., \& Vandierendonck, A. (2008). Aftereffects of response inhibition: Repetition priming or between-trial control adjustments. Journal of Experimental Psychology: Human Perception and Performance, 34, 413-426. 
Verbruggen, F., Logan, G. D., \& Stevens, M. A. (2008). STOP-IT: Windows executable software for the stop-signal paradigm. Behavior Research Methods 40, 479-483.

Verbruggen, F., Schneider, D. W., \& Logan, G. D. (in press). How to stop and change a response: The role of goal activation in multitasking Journal of Experimental Psychology: Human Perception and Performance.
Vince, M. A. (1948). The intermittency of control movements and the psychological refractory period. British Journal of Psychology, 38, 149-157.

Voss, A., \& Voss, J. (2007). Fast-dm: A free program for efficient diffusion model analysis. Behavior Research Methods, 39, 767-775.

Wagenmakers, E. J., van der Maas, H. L. J., \& Grasman, R. (2007). An EZ-diffusion model for response time and accuracy. Psychonomic Bulletin \& Review, 14, 3-22.

\section{Appendix A}

\section{Position Analyses Experiment 1}

To further test the response-strategy adjustment hypothesis, we examined the effect of trial position following the context cues in Experiment 1. Each cue was presented for 4 or 8 trials (see Figure 3). If response-strategy adjustments are made over the course of the first couple of trials, then RT and accuracy in the all context should increase over the first couple of trials whereas RT and accuracy in the none context should decrease over the first couple of trials (this interaction is depicted in the top panels of Figure A1; for a similar idea, see, e.g., Brown \& Steyvers, 2005). By contrast, if subjects immediately make
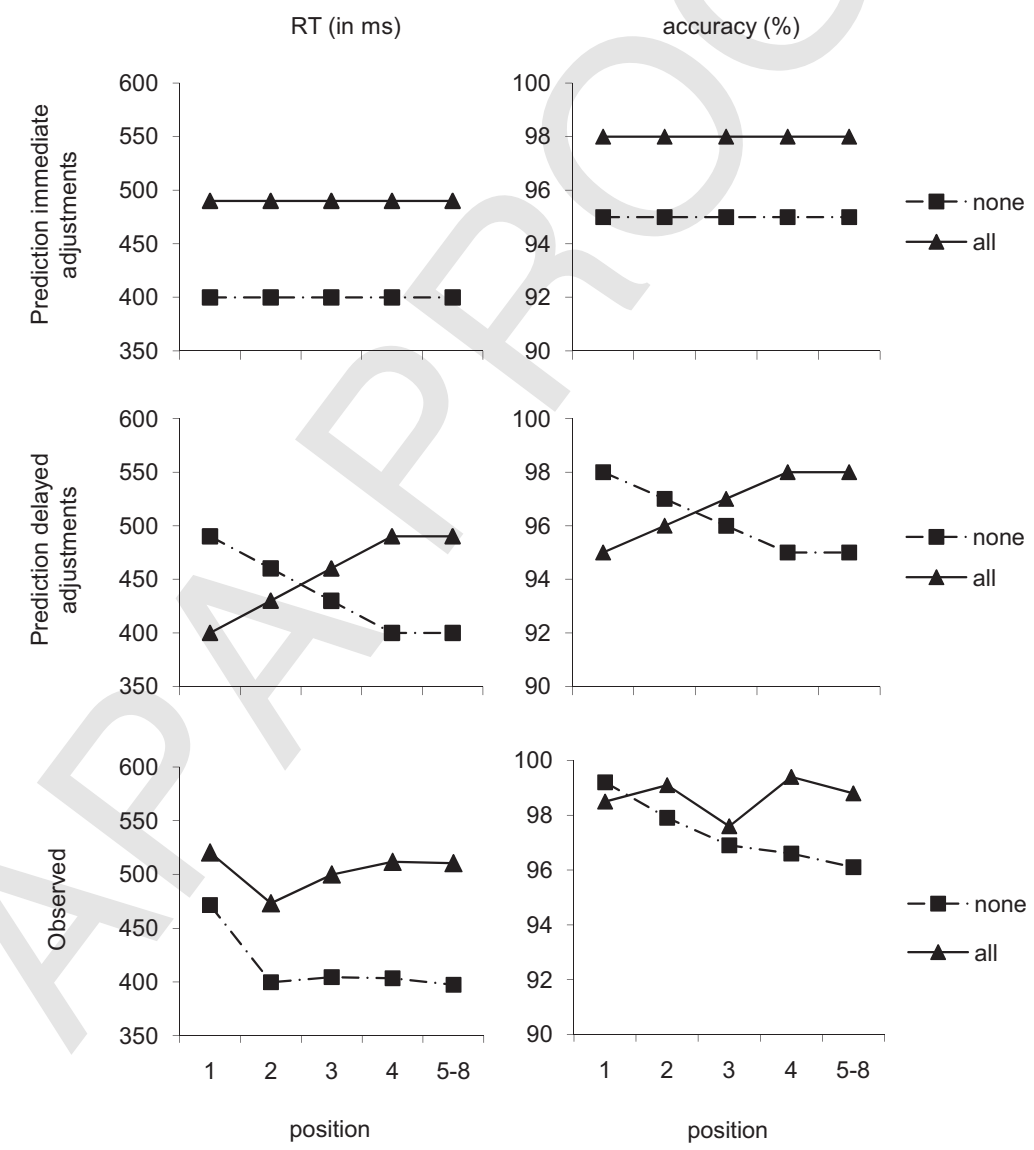

Figure A1. Top and middle panels: Predicted mean RT (left panel) and accuracy (right panel) for each context as a function of the trial position. When subjects make proactive response-strategy adjustments at the beginning of a run of trials, RT and accuracy should not be influenced by trial position; when response-strategy adjustments are made throughout a run of trials, RT and accuracy should be influenced by trial position. Bottom panels: Observed mean RT (left panel) and accuracy (right panel) for each context as a function of the trial position in Experiment 1. 
proactive response-strategy adjustments when the context cue is presented at the start of a run, then there should be no such interaction and the RT and accuracy differences between the none and all contexts should be the same for all positions.

We excluded trials that followed a stop-signal trial or an incorrect no-stop-signal trial (including no-stop-signal trials on which no response was executed) in analyses of go performance. Mean RTs were calculated after removal of go errors. We collapsed across position 5-8 to have sufficient observations in all cells of the data matrix. RTs and accuracy data were analyzed by means of a 2 (context: 'all' vs. 'none') by 5 (position: 1, 2, 3, 4, 5-8) repeated measures ANOVA. We will only focus on the interaction between context and position here. RTs and accuracy scores are depicted in Figure A1.

For RTs, we found a significant interaction between position and context, $F(4,68)=8.0, \operatorname{MSE}=797, p<.001, \eta_{p}^{2}=.32$, suggesting that the difference between the all and none contexts was larger at the end of a run than at the beginning of a run (see Figure A1). Note that RTs were longer on the first trial of a run than on trials 2-8 of a run in the none and all contexts (see Figure A1), suggesting a residual context cue-encoding effect (see, e.g., Logan \& Bundesen, 2003). For the accuracy data, we found an interaction between position and context, $F(4,68)=2.6, M S E=$ $8, p<.05, \eta_{p}^{2}=.13$. As can be seen in Figure A1, accuracy decreased in the none context over the run of trials whereas accuracy was similar for all positions in the all context. Combined, the RTs and the accuracy data suggest that in the all context, subjects made proactive response-strategy adjustments at the beginning of the context (i.e., before the first trial) by increasing the response threshold. By contrast, the accuracy data suggest that in the none context, the response threshold is lowered during a run of trials. In other words, the position analyses suggest that subjects increase the response threshold at the beginning of the all context, whereas they lower the response threshold gradually in the none context.

\section{Appendix B}

\section{Position Analyses Experiment 3}

For run length 2 and run length 4, we also analyzed the effect of position. We excluded trials that followed a stop-signal trial or an incorrect no-stop-signal trial (including no-stop-signal trials on which no response was executed) in analyses of go performance. Mean RTs were calculated after removal of go errors. RTs and accuracy data were analyzed by means of a separate repeated measures ANOVA with context and position as within-subjects factors. We will only focus on the interaction between context and position here. RTs and accuracy scores are depicted in Figure B1. For run length 2, the interaction between context and positions were nonsignificant for RTs and accuracy, both $F$ 's $<1.7$. For run length 4 , we found a significant interaction for RTs, $F(4,68)=$ 10.3, MSE $=1,360, p<.001, \eta_{p}^{2}=.38$. For accuracy, the interaction was not significant, $F<1$. Combined, the RT data and accuracy data once more suggest that subject made proactive response-strategy adjustments at the beginning of the all context.

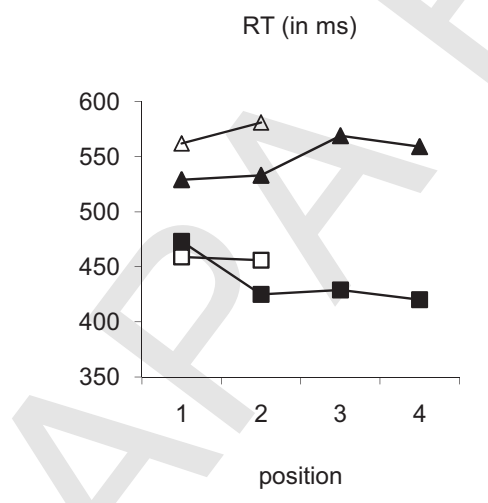

accuracy $(\%)$

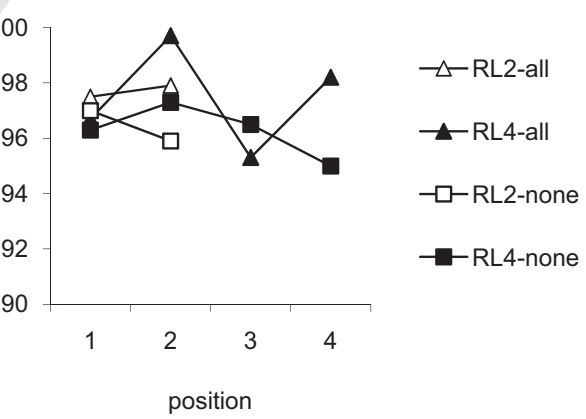

Figure B1. Mean RT (left panel) and accuracy (right panel) for each context and run-length condition (RL2 = run length 2 or RL4 = run length 4 ) as a function of the trial position in Experiment 3. 


\section{Appendix C}

\section{Position Analyses Experiment 5}

We excluded trials that followed a stop-signal trial or an incorrect no-stop-signal trial (including no-stop-signal trials on which
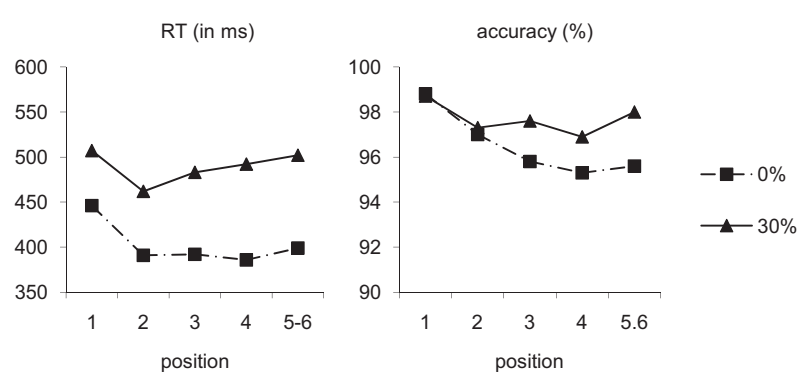

Figure C1. Mean RT (left panel) and accuracy (right panel) for the $0 \%$ and $30 \%$ context in Experiment 5.
RTs were calculated after removal of go errors. RTs and accuracy data were analyzed by means of a separate repeated measures ANOVA with context and position as within-subjects factors. We will only focus on the interaction between context and position here. RTs and accuracy scores are depicted in Figure C1.

For RTs, we found a significant interaction between position and context, $F(4,68)=5.5, M S E=635, p<.01, \eta_{p}^{2}=.24$, suggesting that the difference between the $0 \%$ and $30 \%$ contexts was larger at the end of a run than at the beginning of a run (see Figure $\mathrm{C} 1)$. We did not find such interaction for the accuracy data, $F(4,68)=1.8, M S E=5.4, p>.13, \eta_{p}^{2}=.10$, suggesting that the proactive adjustments were made at the beginning of a run.

Received July 22, 2007 Revision received March 30, 2008 Accepted April 10, 2008 